\title{
An adaptive concurrent multiscale model for concrete based on coupling finite elements
}

\author{
Eduardo A. Rodrigues ${ }^{\mathrm{a}}$, Osvaldo L. Manzoli ${ }^{\mathrm{b}, *}$, Luís A.G. Bitencourt Jr. ${ }^{\mathrm{a}}$, \\ Túlio N. Bittencourt ${ }^{a}$, Marcelo Sánchez ${ }^{\mathrm{c}}$ \\ ${ }^{a}$ University of São Paulo, Av. Prof. Luciano Gualberto, Trav. 3 n. 380, CEP-05508-010 São Paulo-SP, Brazil \\ ${ }^{\mathrm{b}}$ São Paulo State University, UNESP/Bauru, Av. Eng. Luiz Edmundo C. Coube 14-01, CEP-17033-360 Bauru-SP, Brazil \\ ${ }^{\mathrm{c}}$ Texas A\&M University, Zachry Department of Civil Engineering, College Station, TX 77843-3136, USA
}

Received 13 April 2017; received in revised form 26 July 2017; accepted 29 August 2017

Available online 7 September 2017

\begin{abstract}
A new adaptive concurrent multiscale approach for modeling concrete that contemplates two well separated scales (represented by two different meshes) is proposed in this paper. The macroscale stress distribution is used as an indicator to identify critical regions (where the material is prone to degrade) with the explicit aim to enrich these zones with detailed mesoscale material information comprising three basic phases: coarse aggregates, mortar matrix and interfacial transition zone. Thus, the concrete initially idealized as a homogeneous material is gradually replaced and enhanced by a heterogeneous multiphase one. This technique is particularly powerful to handle cases where the region with nonlinear behavior is not easy to anticipate. Furthermore, the proposed approach does not require the definition of a periodic cell (or a RVE), and the meshes from distinct scales are totally independent. The new adaptive mesh technique is based on the use of coupling finite elements to enforce the continuity of displacements between the non-matching meshes associated with the two different scales of analysis. Besides that, mesh fragmentation concepts are incorporated to simulate the crack formation and propagation at the mesoscopic scale, without the need of defining complex and CPU-time demanding crack-tracking algorithms. The strategy is developed integrally within the framework of continuum mechanics, which represents an advantage with respect to other approaches based on discrete traction/separation-law. Numerical examples with complex crack patterns are conducted to validate the proposed multiscale approach. Furthermore, the efficiency and accuracy of the novel technique are compared against full mesoscale and standard concurrent multiscale models, showing excellent results.
\end{abstract}

(c) 2017 Elsevier B.V. All rights reserved.

* Corresponding author.

E-mail address: omanzoli@feb.unesp.br (O.L. Manzoli). 


\section{Introduction}

The process of initiation and propagation of cracks in heterogeneous materials like concrete is a multiscale phenomenon. The local damage on a structural member is the result of physical processes occurring at a lower scale, identified hereafter as the mesoscale that involves: coarse aggregates, mortar matrix and an interfacial transition zone (ITZ). This multiphase meso-level dictates the fracture properties of the material. The properties of the individual mesoscale components (and their mutual interactions) play a critical role in the crack formation and subsequent propagation, as well as in the global mechanical behavior of the concrete [1,2]. In spite of some satisfactory results obtained with macroscopic constitutive models, it seems they are not the best option to assist a reliable understanding of this problem. The main drawback associated with macroscopic models is related to their limitations to include the effects of the mesoscale in the macroscopic response.

The use of multiscale models has been gaining progressively more prominence, particularly when trying to understand better the material trans-scale failure phenomenon [3-13]. In these models, the macroscopic problem is mainly controlled by the mechanical behavior of the material structure (e.g. the degradation of the micro and meso constituents is translated into loss of stiffness and energy dissipation). Hierarchical and concurrent multiscale approaches have been successfully used to model the nonlinear behavior of concrete structures [14]. These methods were initially based on two main concepts: scale separation and representative volume element (RVE) [15-20]. Distinct scale models are sequentially coupled in a continuous interaction between them. Two interacting processes are generally involved in this type of analysis: (i) the localization process, in which the coarser-scale response is transferred to the finer-scale material model to evaluate the behavior of the material structure, and (ii) the homogenization process, where the information from the finer structure is upscaled (to feed the constitutive equations related to the coarser-scale), to solve the macroscale problem [21]. This interaction characterizes the trans-scale process, where the nonlinear behavior occurring in the finer-scale controls the coarser-scale response, at the same time the boundary conditions at the macroscale affect the failure process taking place at the finer-scale. Usually this technique is known as $F E^{2}$ multiscale method because there is a finite element (FE) model inside another FE model [22]. Therefore, for each integration point of the macroscopic (global) FE mesh, a RVE analysis is conducted, and the homogenized response is returned up to the macroscale model.

One of the main challenges associated with the FE modeling of heterogeneous materials (e.g. concrete) incorporating mesoscale effects is to accurately represent the internal material structure using the current computer power. For example, analyses involving small concrete specimens (i.e. reduce scale experiments or similar), have been conducted based on a purely mesoscale approach to represent the material structure [2,23-27]. The excellent results obtained in these studies show that mesoscopic models are very useful for studying the influence of the concrete internal-structure (i.e. coarse aggregates, mortar matrix and the ITZ) on the macroscopic response. However, the very fine mesh necessary to conduct this type of analysis increases dramatically the computational effort (i.e. CPU time and memory requirements), which makes practically prohibitive the modeling of engineering problems using a pure mesoscale approach.

In the concurrent multiscale model the structure is generally divided into two main subdomains: (i) "critical" zones (i.e. regions where inelastic processes are anticipated), and (ii) "undamage" zones. The explicit representation of the concrete mesoscale circumscribes to those sub-domains where the nonlinear behavior is expected (i.e. critical regions). Both scales are solved simultaneously, resulting in a strong coupling between them $[17,28,14]$. An advantage of this method is that the definition of a RVE is not necessary. However, an efficient scheme to couple the non-matching meshes is needed to enforce global equilibrium and displacements compatibility between the subdomains, aspect that can be very challenging. Another advantage of this kind of approach is that the failure of the material can be explicitly simulated. Furthermore, accurate results in terms of both, crack initiation and propagation can be achieved. However, as pointed out by [17], the subdomains not only differ in terms of mesh refinement (i.e. a much finer mesh is required to represent the mesoscale), but also in terms of constitutive modeling (i.e. the behavior of each phase of the proposed material needs to be properly reproduced).

The finer-scale subdomains in concurrent multiscale models can be defined a priori to reduce the numerical effort and memory demand. These models are called explicit direct multiscale models and the most difficult task is to anticipate the regions that will present a nonlinear behavior to introduce there the refined scale mesh. To circumvent this problem, a number of adaptive multiscale models have been proposed [25,29-33]. In these models, the finer-scale region is not pre-defined but incorporated during the nonlinear simulation. The adaptive schemes are not new and they are not restricted to multiscale techniques. Mesh adaptation procedures, for example the $h$ - $p$-adaptation method, has 
been successfully applied to minimize the computational cost and the discretization error in computational mechanics [34].

In the multiscale context, the adaptive procedure can be used to enrich the modeling or to improve the computational efficiency. Based on appropriate adaptive indicators, the coarser-scale can be replaced by the finerscale in the critical regions only. In general, these adaptive multiscale approaches are formulated using two or even three scales by using the concept of non-overlapping subdomains together with coexisting periodic cells or RVE [29]. Those subdomains where the adopted criterion is violated are replaced by the corresponding periodic cell or RVE. As a consequence of the adaptation process, several new meshes will appear in the critical regions and a coupling technique is needed to connect these independent subdomains. The Lagrange multipliers technique [35-37] is usually adopted to ensure the correct continuity and compatibility conditions at the non-matching interfaces between them. This technique introduces extra unknowns to the system of equations associated with the Lagrange multipliers that increases the numerical effort and memory demand. Moreover, as a consequence of the coexisting periodic cells or RVEs, regular subdomains need to be defined.

This paper proposes an alternative adaptive concurrent multiscale model for concrete. In this model the material is represented by two well separated scales in a concurrent manner. The macroscale considers the concrete as a homogeneous single-phase material (i.e. the internal structure is disregarded at this level), while the mesoscale contemplates the typical three phases observed in concrete, namely: coarse aggregates, mortar matrix and the ITZ. During the numerical analysis, the homogenized macroscale FE mesh is upgraded with information from the mesoscale in those critical regions identified by a macroscopic indicator. Consequently, the macroscopic mesh is automatically replaced by a mesoscopic one in those zones where the nonlinear behavior is imminent. The main novelty of the proposed technique is the introduction of coupling finite elements proposed by [38] for connecting nonmatching meshes from macro and mesoscale regions. This technique ensures the continuity of displacements between independent and concurrent meshes (i.e. macroscale and mesoscale meshes), without increasing the total number of degrees of freedom of the problem. Moreover, periodic cells or RVEs are not required in this method. All the nonlinear behavior of the material is explicitly ascribed to the mesoscopic level, applying the approach proposed by Rodrigues et al. [26] based on the mesh fragmentation technique (MFT) proposed by Manzoli and collaborators [39,40]. Thus, the analyses are conducted integrally in the context of the continuum mechanics. Furthermore, the formation and propagation of complex and arbitrary cracks can be simulated without the need of tracking algorithms.

The remainder of this paper is organized as follows. An overview and the main aspects of the proposed adaptive concurrent multiscale technique are presented in Section 2. Section 3 details the main ingredients of the mesoscopic model used to simulate the crack formation and propagation based on the MFT. In Section 4 three numerical analyses are conducted in order to validate the proposed approach. Finally, a summary and the main conclusions are presented in Section 5.

\section{Adaptive concurrent multiscale model for concrete}

This section presents the proposed adaptive concurrent multiscale model for concrete.

\subsection{An overview}

Let us consider the problem depicted in Fig. 1(a), in which a prescribed displacement field is imposed on a concrete specimen. The first step to capture the crack propagation process consists in the discretization of the problem in two (initially independent) FE meshes. The coarse mesh is adopted to represent the material behavior at the macroscale (Fig. 1(b)), which is assumed to be linear elastic and based on effective (or homogenized) material properties. The fine mesh (i.e. Fig. 1(c)) represents the mesoscale with the associated heterogeneities of the concrete. As it can be seen in Fig. 1(d), the idea is to create two layers with independent meshes (associated with the two material scales), which will be combined based on the stress-dependent evolution laws to construct the concurrent multiscale model.

Thus, based on the macro and mesoscopic meshes discussed above, the proposed adaptive concurrent multiscale model can be described according to the flowchart shown in Fig. 2 consisting of the following main steps:

1. During the first loading step of the analysis, the entire domain is represented by the macroscopic mesh and a linear elastic solution is obtained. 


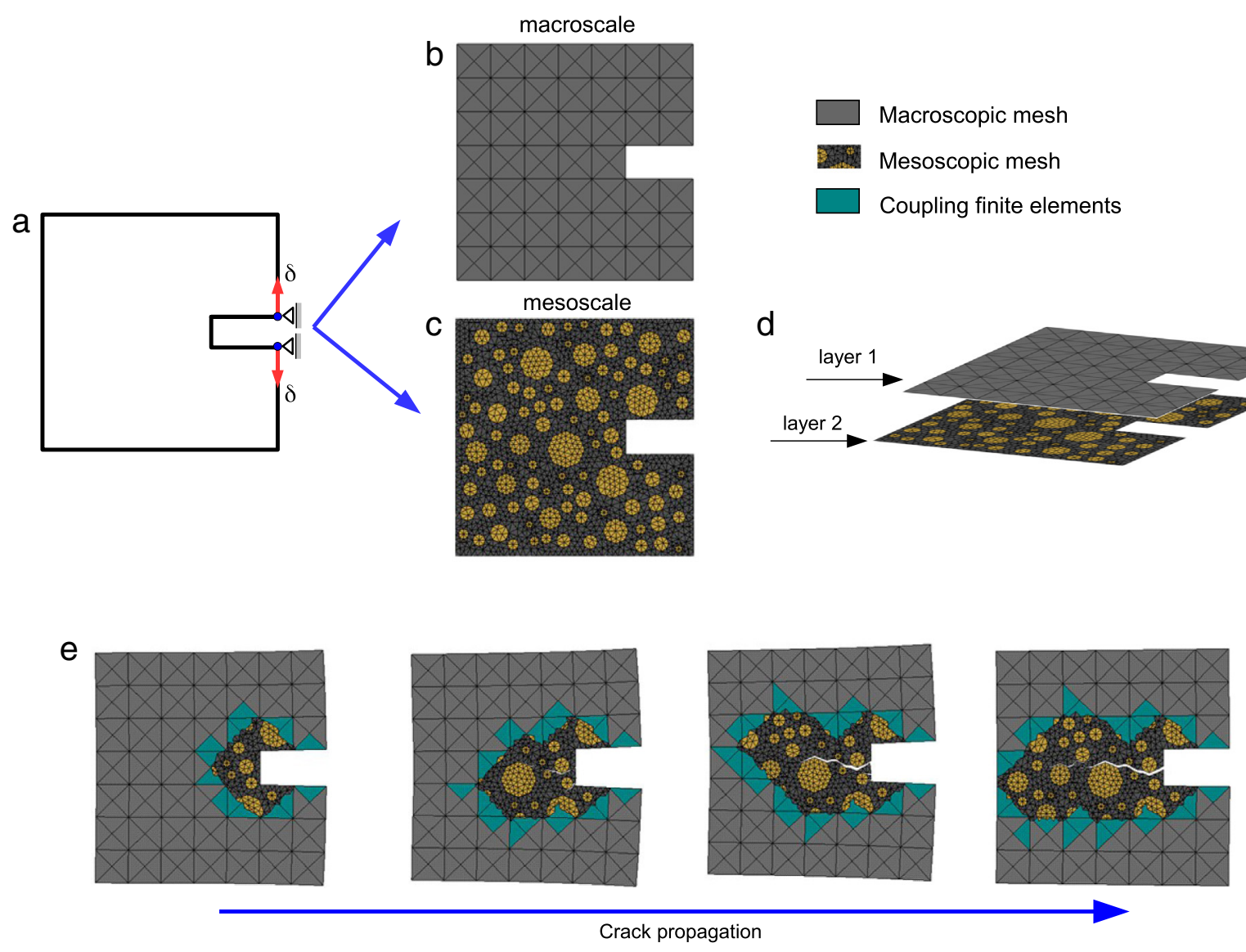

Fig. 1. Proposed adaptive concurrent multiscale model.

2. During the second loading stage (and at the beginning of each subsequent loading step), an appropriate adaptive scale-change criterion is checked at each integration point of the macroscopic elements (see Section 2.2 for details about the adopted adaptive mesh criterion).

3. If at any integration point of a macroscopic element, the adaptive scale-change criterion is violated, this element is deactivated and replaced by the corresponding mesoscopic mesh that has at least one node that belongs to the domain of the macroscopic element that is being deactivated.

4. To enforce the equilibrium and ensure the displacement compatibility between the non-matching meshes from the distinct scales, coupling elements are introduced to connect macro and mesoscopic meshes (Fig. 1(e)).

5. Finally, once the concurrent multiscale model (at a given loading step) is completed, the converged solution is obtained and the maximum positive principal stress $\left(\sigma_{\max }\right)$ associated with each macroscopic element is stored to be used in the next step to check the scale-change criterion.

The mesoscale mesh can be generated either prior to run the case (as it was described above) or, as the analysis evolves, the new mesh can be generated and inserted in those zones where it is necessary to include it. The first option was selected in this work to save the computational time associated with the interruptions required in the second option for the generation and upgrading of the mesh in the selected zones. The procedure adopted here looks like more efficient in terms of processing time, at least for the size of the problems considered in this research. However, for problems involving a large number of degrees of freedom (i.e. large scale engineering structures), it is possible that an adaptive strategy to generate the new meshes as the loading process progresses may be more efficient. This is an interesting research area to be explored in the future. 


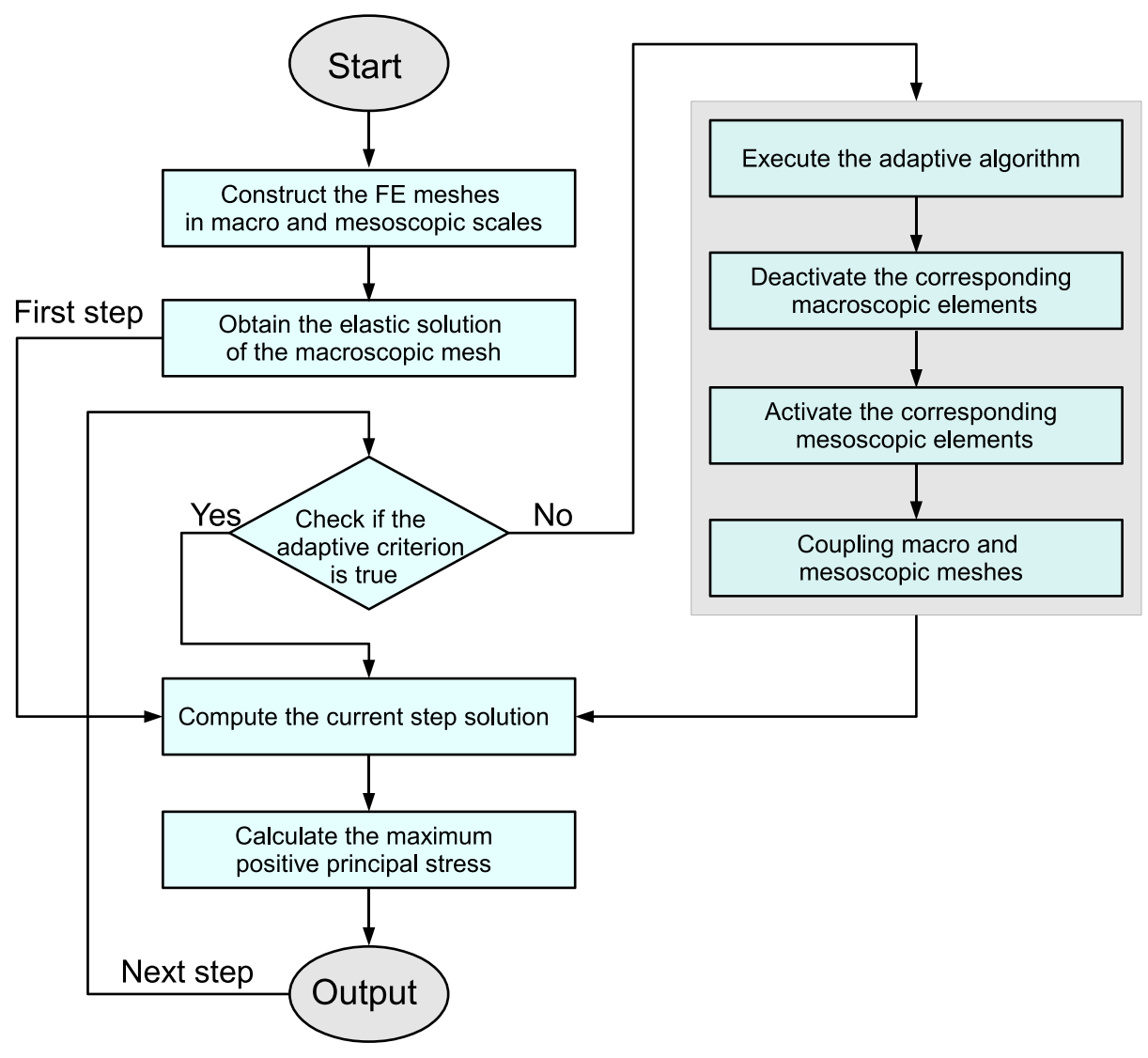

Fig. 2. Flowchart of the proposed adaptive concurrent multiscale model.

An advantage of the proposed approach is that there are no requirements with respect to mesh alignment, it is not necessary to enforce coincidence of nodes between macro and meso discretizations.

A possible drawback of this method is that eventual mesh overlaps between macro and meso discretizations may introduce spurious change in the global stiffness matrix. However, this problem can be certainly minimized by increasing the mesh refinement of the mesoscopic model.

\subsection{Adaptive mesh criterion}

An adaptive scale-change criterion is defined to update the model in the critical regions from the macroscale to the mesoscale to capture the crack propagation process considering the material internal structure. Therefore, from the second loading step onward (see flowchart in Fig. 2), the following equation is checked:

$$
F\left(\sigma_{\text {max }}, F t_{\text {adap }}\right)=\sigma_{\text {max }}-F t_{\text {adap }}<0
$$

where the critical regions at macroscale domain are defined by the difference between the maximum positive principal stress $\left(\sigma_{\max }\right)$ calculated in the previous step and the adaptive mesh predictor $\left(F t_{\text {adap }}\right)$. In the context of this work, the adaptive mesh predictor will be defined as a function of the concrete tensile strength.

The damage process tends to initiate in the ITZ. Therefore, the adaptive indicator (based on the maximum positive principal stress of the macroscopic mesh) should preferable assume values that are close to the tensile strength of the ITZ or between the ITZ and matrix tensile strength. On one hand, if it is assumed that the adaptive indicator is smaller than the ITZ strength, the mesoscale mesh will be activated well before any failure occurs. This creates a very large region of the macroscopic mesh to be replaced by the mesoscopic one. On the other hand, if higher values than the 
Matrix (bulk) elements

Matrix-matrix interface elements
Aggregate elements

ITZ interface elements

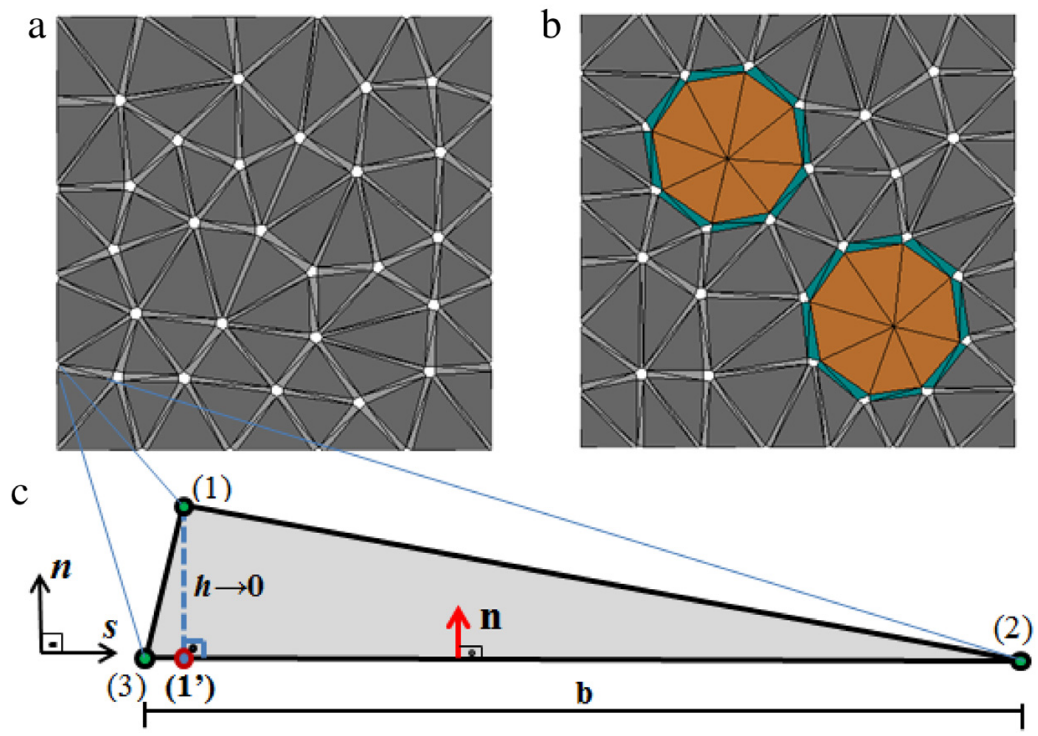

Fig. 3. Mesoscopic model based on the mesh fragmentation technique (MFT): (a) fragmented mesh, (b) matrix-aggregate and matrix-matrix interface representations and (c) finite element with high aspect ratio.

tensile strength of the matrix are assumed for the adaptive indicator, the mesoscopic mesh may be activated in a very restricted region. However, the mesoscale damage process can be in a very advanced stage, which may negatively affect the simulation of actual failure process.

Note that the adapted adaptive criterion is based on the previous stress state of the macroscopic finite elements only. However, other criteria to transfer the macroscale to the mesoscale domain could be adopted. For example, as proposed by [25], the criterion can be based on a combination of the maximum principal positive stress for the macroscale with the spatial distribution of damage on the mesoscopic mesh that is already activated.

\subsection{Mesoscopic model}

The mesoscopic scale is represented by the approach proposed in [26] based on the fragmentation technique [40,39], which consists in the insertion of FE with high aspect ratio HAR [41] between all the bulk finite elements. These interface elements play a critical role to model the mesoscale behavior because they concentrate all the non-linear behavior of the material, which allows simulating the crack propagation process on the matrix and also between the bulk matrix and the aggregate elements (i.e. representing the complex behavior of the ITZ). Fig. 3(a) shows the fragmented mesh and Fig. 3(b) illustrates the two embedded aggregates with the surrounding ITZ interface elements. To keep this figure readable, the interface elements are much bigger than the ones actually implemented. Fig. 3(c) presents a HAR element.

The basic formulation of the interface finite element and the continuum damage model adopted to describe the material behavior are described in the following sections. More details about the MFT and the mesoscale model can be found elsewhere (e.g. $[40,39,26])$.

The damage criterion of the interface elements defines the crack initiation in the mesoscopic model. This damage criterion is based on the tensile stress normal to the interface elements (see Section 3.1.1) indicating damage evolution and the corresponding dissipation process when the tensile stress reaches the tensile strength of the concrete. To obtain a good correspondence with the damage criterion of the mesoscopic model, the adaptive scale-change indicator is based on the maximum positive principal stress of the macroscopic mesh. 

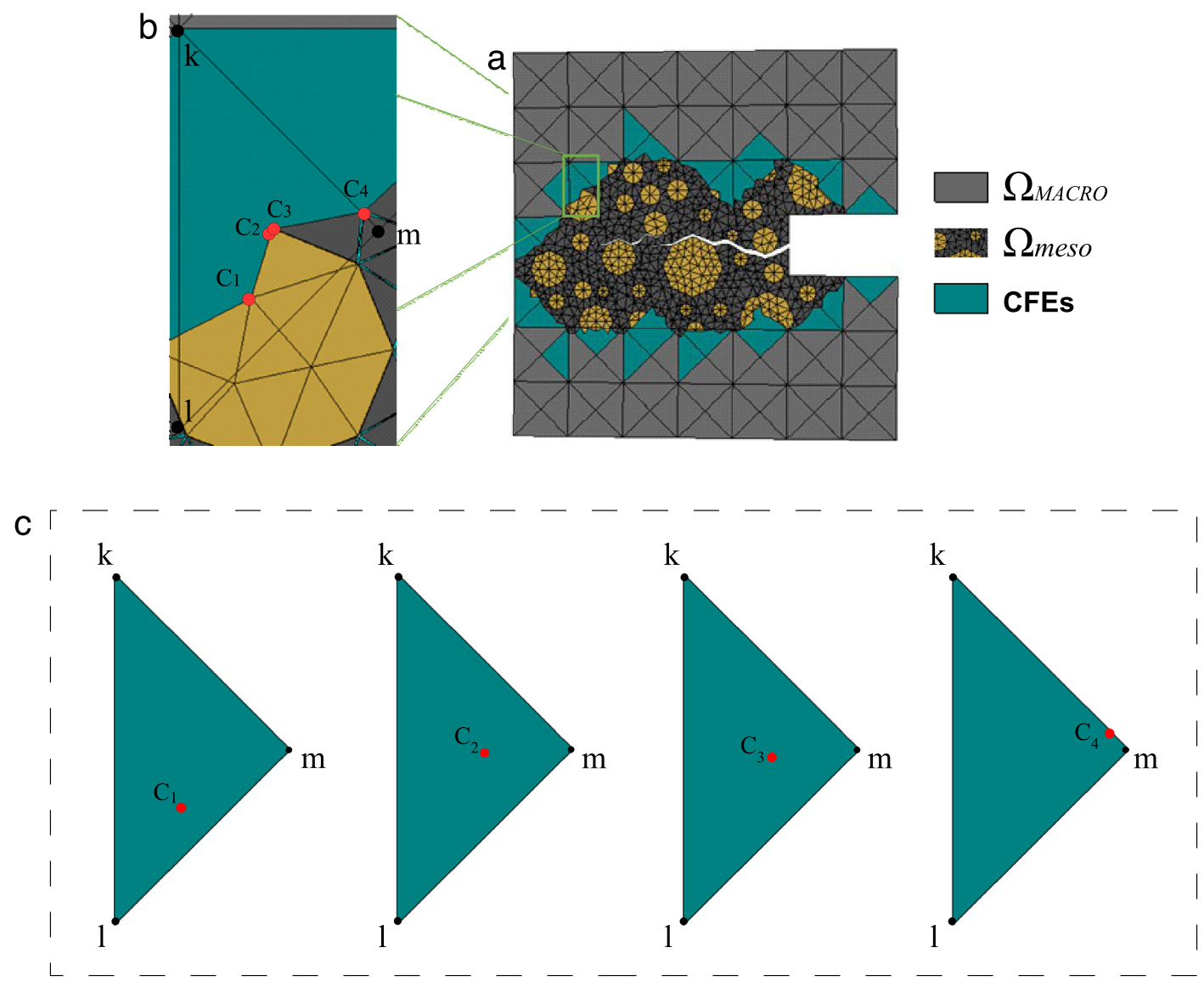

Fig. 4. Coupling technique based on the use of coupling finite elements to connect the non-matching meshes of the macroscopic $\left(\Omega_{M A C R O}\right)$ and mesoscopic ( $\Omega_{\text {meso }}$ ) domains: (a) insertion of CFEs at a loading step, (b) details of the floating nodes $\left(C_{1}, C_{2}, C_{3}\right.$ and $\left.C_{4}\right)$ on the domain of the base element $F E^{\Omega_{M A C R O}}=\{k, l, m\}$ and (c) CFEs inserted in the mesh.

\subsection{Coupling the macroscopic and mesoscopic meshes}

The strategy proposed by [38] for coupling non-matching meshes is adopted in this paper to couple the concurrent macro and mesoscopic meshes at each loading step during the nonlinear regime. This technique is based on the use of coupling finite elements (CFEs), which can be derived from a standard isoparametric FE with an additional node, called coupling node $\left(C_{\text {node }}\right)$.

Fig. 4(a) shows the insertion of CFEs (i.e. green elements) to couple the domain of the macroscopic $\left(\Omega_{M A C R O}\right)$ and mesoscopic $\left(\Omega_{\text {meso }}\right.$ ) meshes at the last loading step presented in Fig. 1. A four-noded triangular coupling FE is introduced for each "floating node" (also known as "loose node", it corresponds to those nodes of the mesoscale mesh that do not have a corresponding one in the macroscale mesh) of the mesoscopic domain. Fig. 4(b) depicts the threenoded triangular finite element $F E^{\Omega_{M A C R O}}=\{k, l, m\}$ that is the base element to construct the following four coupling finite elements: $C F E_{1}=\left\{k, l, m, C_{1}\right\}, C F E_{2}=\left\{k, l, m, C_{2}\right\}, C F E_{3}=\left\{k, l, m, C_{3}\right\}$ and $C F E_{4}=\left\{k, l, m, C_{4}\right\}$ (Fig. 4(c)). Each CFE has three nodes that are identical to an underlying FE of the macroscopic mesh $\left(\Omega_{M A C R O}\right)$ and an extra node coinciding with the floating node belonging to the mesoscopic mesh $\left(\Omega_{\text {meso }}\right)$, called coupling node (i.e. $C_{1}, C_{2}, C_{3}$ and $C_{4}$ ).

Fig. 4(c), considers a CFE derived from an isoparametric element with domain $\Omega_{e}$, containing $n n$ nodes, shape functions $\bar{N}_{i}(i=1: n n)$ and an additional node, $n n+1$, located on the $\mathbf{X}_{C} \in \Omega_{e}$, which has the same coordinates of the $C_{\text {node }}$. A relative displacement $(\llbracket \mathbf{U} \rrbracket)$ can be defined as the difference between the displacements of the material point $\mathbf{X}_{C}$, evaluated using the shape functions of the underlying FE and the actual displacements of the $C_{\text {node }}$. 
[38] defines the reaction force associated with these relative displacements as:

$$
\mathbf{F}(\llbracket \mathbf{U} \rrbracket)=\mathbf{C} \llbracket \mathbf{U} \rrbracket=\mathbf{C} \underbrace{\sum_{i}^{n n} \bar{N}_{i}\left(\mathbf{X}_{C}\right) \mathbf{D}_{i}-\mathbf{D}_{n n+1}}_{\llbracket \mathbf{U} \rrbracket}
$$

where $\mathbf{D}_{i}=(i=1: n n)$ is the nodal displacement vector of the isoparametric FE used as base element, $\mathbf{D}_{n n+1}$ is the displacement vector of the $C_{\text {node }}$ and $\mathbf{C}$ is the matrix that stores the constant elastic components. High values of these constant $(\tilde{C})$ can be assumed to ensure the displacement compatibility of the two non-matching meshes:

$$
\mathbf{C}=\left[\begin{array}{ccc}
\tilde{C} & 0 & 0 \\
0 & \tilde{C} & 0 \\
0 & 0 & \tilde{C}
\end{array}\right]
$$

Note that in Eq. (2) the high elastic value assigned to $(\tilde{C})$ plays the role of a penalty variable in relation to the relative displacement $(\llbracket \mathbf{U} \rrbracket)$. The objective is to 'anchor' the 'floating' elements to the macroscale mesh. Since the equilibrium condition states that the reaction force must be bounded, the relative displacement shall tend to zero.

Eq. (2) can be rewritten as:

$$
\mathbf{F}(\llbracket \mathbf{U} \rrbracket)=\underbrace{\mathbf{C} \mathbf{B}_{e} \mathbf{D}_{e}}_{\llbracket \mathbf{U} \rrbracket}
$$

where $\mathbf{B}_{e}$ is expressed as:

$$
\mathbf{B}_{e}=\left[\begin{array}{lllll}
\overline{\mathbf{N}}_{1}\left(\mathbf{X}_{c}\right) & \overline{\mathbf{N}}_{2}\left(\mathbf{X}_{c}\right) & \ldots & \overline{\mathbf{N}}_{n n}\left(\mathbf{X}_{c}\right) & -\mathbf{I}
\end{array}\right]
$$

$\mathbf{I}$ being the identity matrix. The internal force vector $\mathbf{F}_{e, \Omega_{C F E}}^{\mathrm{int}}$ and the stiffness matrix $\mathbf{K}_{e, \Omega_{C F E}}$ of the CFE can be defined as:

$$
\mathbf{F}_{e, \Omega_{C F E}}^{\text {int }}=\mathbf{B}_{e}^{T} \mathbf{F}(\llbracket \mathbf{U} \rrbracket)=\mathbf{B}_{e}^{T} \mathbf{C B}_{e} \mathbf{D}_{e}
$$

and

$$
\mathbf{K}_{e, \Omega_{C F E}}=\frac{F_{e, \Omega_{C F E}}^{\mathrm{int}}}{\partial \mathbf{D}_{e}}=\mathbf{B}_{e}^{T} \mathbf{C B}_{e}
$$

The global internal force $\left(\mathbf{F}^{\text {int }}\right)$ vector and the stiffness matrix $(\mathbf{K})$ can then be written as:

$$
\mathbf{F}^{\mathrm{int}}=\underbrace{\mathrm{A}_{e=1}^{\mathrm{nel} \Omega_{M A C R O}} \mathbf{F}_{e, \Omega_{M A C R O}}^{\mathrm{int}}}_{\text {macroscopic elements }}+\underbrace{\mathrm{A}_{e=1}^{\mathrm{nel} \Omega_{\text {meso }}} \mathbf{F}_{e, \Omega_{\text {Mmeso }}}^{\mathrm{int}}}_{\text {mesoscopic elements }}+\underbrace{\mathrm{A}_{e=1}^{\mathrm{nel} \Omega_{C}} \mathbf{F}_{e, \Omega_{C F E}}^{\mathrm{int}}}_{\text {coupling elements }}
$$

and

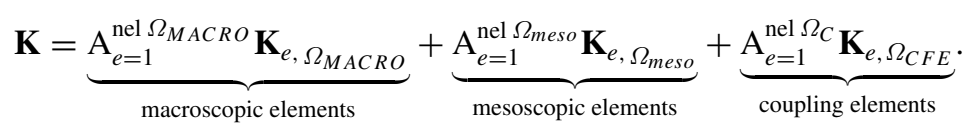

where $A$ stands for the FE assembly operator. The first and second terms of Eqs. (8) and (9) are related to the subdomains $\Omega_{M A C R O}$ and $\Omega_{\text {meso }}$, respectively, and the third term is associated with the incorporation of CFEs in the model. Note that the second and third terms are null during the elastic regime.

The procedure described above is repeated for all the loading steps according to the adaptive scale change criterion defined by Eq. (1). The coupling finite elements are generated during the preprocessing stage. Therefore, the data for coupling all the potential boundary nodes belonging to the mesoscopic mesh is stored in the input file, these elements being activated and deactivated according to the stress field distribution at each loading step. An important advantage of the proposed technique is that macroscopic and mesoscopic meshes are completely independent. In this way, aligned meshes or even pre-defined regular subdomain, as required by other well-known methods [14,25,33], are not necessary here. Any material overlap associated with the connection between the two meshes can be minimized by reducing the size of the mesoscale mesh (Fig. 4(b)). 


\section{Mathematical and numerical framework}

This section presents the main components related to the interface finite element selected in this work to describe the mesoscale, as well as the adopted constitutive mechanical model and the algorithm adopted for its numerical implementation.

\subsection{Interface finite element with high aspect ratio}

Let us consider the three-noded triangular FE depicted in Fig. 3(c), with height $h$, given by the distance between the node (1) and its projection on the element base, $\left(1^{\prime}\right)$; as well as, the local coordinate system $(n, s)$, oriented such that axis $s$ coincides with the orientation of the base element.

Following the standard FE approximation, the strain tensor at any point of the element can be approximated by:

$$
\boldsymbol{\varepsilon}=\tilde{\boldsymbol{\varepsilon}}+\hat{\boldsymbol{\varepsilon}}=\frac{1}{b}\left[\begin{array}{ccc}
0 & \frac{1}{2}\left(u_{n}^{(3)}-u_{n}^{(2)}\right) & 0 \\
\frac{1}{2}\left(u_{n}^{(3)}-u_{n}^{(2)}\right) & \left(u_{s}^{(3)}-u_{s}^{(2)}\right) & 0 \\
0 & 0 & 0
\end{array}\right]+\frac{1}{h}\left[\begin{array}{ccc}
\llbracket u \rrbracket_{n} & \frac{1}{2} \llbracket u \rrbracket_{s} & 0 \\
\frac{1}{2} \llbracket u \rrbracket_{s} & 0 & 0 \\
0 & 0 & 0
\end{array}\right]
$$

where the part $\hat{\boldsymbol{\varepsilon}}$ collects all the components of the strain tensor which depends on $h$ and $\tilde{\boldsymbol{\varepsilon}}$ contains the rest of the components, $u_{n}^{(i)}$ and $u_{s}^{(i)}$ are the displacement components of the node (i); and $\llbracket u \rrbracket_{n}$ and $\llbracket u \rrbracket_{s}$ are the components of the relative displacement between node (1) and the point corresponding to its projection on the element base $\left(1^{\prime}\right)$.

In the case of a general coordinate system, it is possible to write the strain part associated with $h$ as:

$$
\hat{\boldsymbol{\varepsilon}}=\frac{1}{h}(\mathbf{n} \otimes \llbracket \mathbf{u} \rrbracket)^{S}
$$

where $(\bullet)^{S}$ refers to the symmetric part of $(\bullet), \mathbf{n}$ is the unit vector normal to the element base, $\otimes$ denotes a dyadic product and $\llbracket \mathbf{u} \rrbracket$ is a vector that stores the components of the relative displacement. Therefore, the total strain tensor given by Eq. (10) becomes:

$$
\boldsymbol{\varepsilon}=\tilde{\boldsymbol{\varepsilon}}+\underbrace{\frac{1}{h}(\mathbf{n} \otimes \llbracket \mathbf{u} \rrbracket)^{S}}_{\hat{\boldsymbol{\varepsilon}}} .
$$

Note that, when the height $h$ tends to zero, the strain component $\tilde{\boldsymbol{\varepsilon}}$ remains bounded while the component $\hat{\boldsymbol{\varepsilon}}$ is no longer bounded. Therefore, in the limit situation of $h$ tending to zero, the element strains are related almost exclusively to the relative displacement between node (1) and its projection on the element base, (1'), implying that node (1) and its projection $\left(1^{\prime}\right)$ tend to the same material point. Consequently, the relative displacement $\llbracket \mathbf{u} \rrbracket$ becomes a measure of the displacement discontinuity (strong discontinuity). The structure of the strain field in Eq. (12) corresponds to the typical kinematics of the Continuum Strong Discontinuity Approach (CSDA) [42-44]. As a consequence, the analyses can be performed integrally in the context of the continuum mechanics, which represents a clear advantage of this method.

\subsubsection{Tensile damage model with IMPL-EX}

In this work, a tension damage constitutive relation between stress and strain is proposed to describe the crack formation and propagation processes. The nonlinear behavior of this model is described by means of a single scalar damage variable, $d$, which ranges from zero (for the case of undamaged material) to one (for the case of a completely damaged concrete). The model is based on the effective stress field, $\overline{\boldsymbol{\sigma}}=\mathbf{C}: \boldsymbol{\varepsilon}$, and its damage criterion is defined as a function of the stress component normal to the base of the element $\left(\bar{\sigma}_{n n}\right)$. Thus, if the normal stress component is greater than zero $\left(\bar{\sigma}_{n n}>0\right)$, the current stress tensor is calculated after applying the damage variable to degrade all the components of the effective stress tensor as,

$$
\sigma= \begin{cases}(1-d) \bar{\sigma} & \text { if } \bar{\sigma}_{n n}>0 \\ \bar{\sigma} & \text { if } \bar{\sigma}_{n n} \leq 0\end{cases}
$$


Table 1

IMPL-EX integration scheme for the continuum tensile damage model.

\begin{tabular}{|c|c|c|}
\hline Evaluate the effective stress tensor & $\overline{\boldsymbol{\sigma}}_{n+1}=\mathbf{C}: \boldsymbol{\varepsilon}_{n+1}$ & (14) \\
\hline $\begin{array}{l}\text { Damage criterion and the implicit calculation stage of the } \\
\text { strain-like variable }\end{array}$ & $\begin{array}{ccl}F_{n+1} & = & \bar{\sigma}_{n n}(n+1) \\
& \text { if true } & r_{n+1}=r_{n} \leq 0 \\
& \text { else } & r_{n+1}=\bar{\sigma}_{n n} \\
& & \end{array}$ & (15) \\
\hline Computation of the linear extrapolated internal variable & $\begin{array}{l}\tilde{r}_{n+1}=r_{n}+\frac{r_{n}-r_{n-1}}{\Delta t_{n}} \Delta t_{n+1} \\
\Delta t_{n}=t_{n}-t_{n-1} ; \Delta t_{n+1}=t_{n+1}-t_{n}\end{array}$ & (16) \\
\hline $\begin{array}{l}\text { Calculate the damage variable in terms of the extrapolated } \\
\text { strain-like variable }\end{array}$ & $\tilde{d}_{n+1}=1-\frac{f_{t}}{\tilde{r}_{n+1}} e^{\frac{f_{t}^{2}}{E G_{f}} h\left(1-\frac{\tilde{r}_{n+1}}{f_{t}}\right)}$ & (17) \\
\hline Evaluate the nominal stress tensor & $\tilde{\boldsymbol{\sigma}}_{n+1}=\left\{\begin{array}{lll}\left(1-\tilde{d}_{n+1}\right) \overline{\boldsymbol{\sigma}}_{n+1} & \text { if } & \bar{\sigma}_{n n_{(n)}}>0 \\
\overline{\boldsymbol{\sigma}}_{n+1} & \text { if } & \bar{\sigma}_{n n_{(n)}} \leq 0\end{array}\right.$ & (18) \\
\hline Compute the effective algorithmic tangent operator & $\tilde{\mathbf{C}}_{n+1}^{\text {tang }}=\frac{\partial \tilde{\boldsymbol{\sigma}}_{n+1}}{\partial \boldsymbol{\varepsilon}_{n+1}}=\left\{\begin{array}{lll}\left(1-\tilde{d}_{n+1}\right) \mathbf{C} & \text { if } & \bar{\sigma}_{n n}(n) \\
\mathbf{C} & \text { if } & \bar{\sigma}_{n n}>0 \\
(n) & \leq 0\end{array}\right.$ & (19) \\
\hline
\end{tabular}

In principle, any stress integration algorithm could be adopted to update the stresses. The implicit-explicit (IMPLEX) integration scheme $[45,46]$ was adopted in this work. This scheme has been proved as very efficient and robust to integrate the constitutive tensile damage mode adopted in this work. It has also assisted to circumvent possible numerical instability and convergence issues associated with the complex problem of the formation and propagation of multiple (and simultaneous) cracks in the nonlinear zone of the material.

Table 1 shows the IMPL-EX scheme adopted to integrate the constitutive tensile damage model. In the current pseudo-time step, $t_{n+1}$ and given the strain tensor, $\boldsymbol{\varepsilon}_{n+1}$, the effective stress tensor, $\overline{\boldsymbol{\sigma}}_{n+1}$, is obtained by Eq. (14), where $\mathbf{C}$ is fourth order elastic tensor. In Eq. (15) the current strain-like variable, $r_{n+1}$, is evaluated implicitly according to the damage criterion and saved as a history variable to be used in the next time step. Note that, the strain-like variable is updated (assuming the current component value $\bar{\sigma}_{n n_{(n+1)}}$ ) when the damage criterion condition is violated only.

Once the implicit stage is completed, an explicit linear extrapolation of the strain-like variable, $\tilde{r}_{n+1}$, is performed using the implicit values ( $r_{n}$ and $r_{n-1}$ ) obtained in the previously time steps, $t_{n-1}$ and $t_{n}$, according to Eq. (16). Therefore, the damage variable, $d_{n+1}$, is calculated in terms of the actual extrapolated strain-like variable using Eq. (17), where $f_{t}$ is the material tensile strength, $E$ is the Young's modulus and $G_{f}$ is the fracture energy of the material. The damage variable is then used to compute the nominal stress tensor, $\tilde{\boldsymbol{\sigma}}_{n+1}$, in Eq. (18). The corresponding algorithmic tangent operator is given by Eq. (19).

\section{Numerical model application}

Three examples are analyzed to validate and demonstrate the effectiveness of the proposed multiscale model. Three types of numerical simulations are considered:

- AMS - Adaptive Multiscale Simulation: corresponds to a numerical study based on the proposed adaptive concurrent multiscale model incorporating CFEs.

- DMS - Direct Multiscale Simulation: corresponds to a numerical simulation adopting the standard concurrent multiscale model.

- DNS-M - Direct Numerical Simulation in Mesoscale: corresponds to an analysis adopting a mesoscopic mesh for the whole domain of the problem.

\subsection{Uniaxial tension test}

In this benchmark a concrete sample under plane stress state with an out-of-plane thickness of $100 \mathrm{~mm}$ is subjected to tension. Two numerical analyses were conducted to compare the results obtained with the proposed multiscale 
a

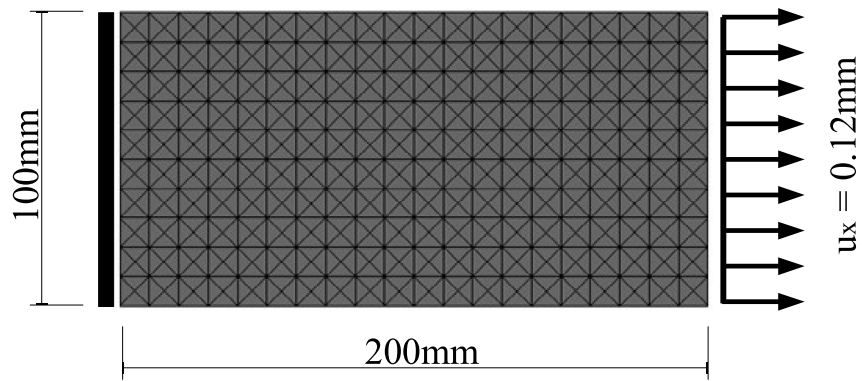

b

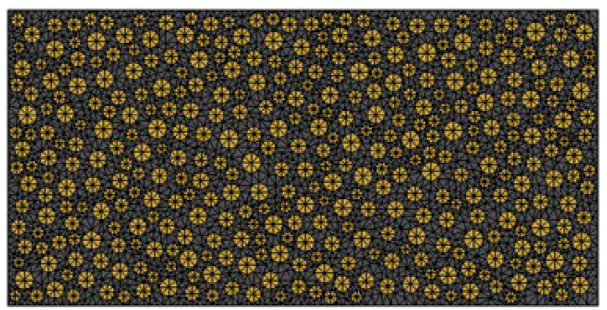

Fig. 5. Concrete sample under uniaxial loading: (a) test setup and macroscopic mesh; and (b) mesoscopic mesh.

Table 2

Material parameters for the uniaxial tension test.

\begin{tabular}{llllll}
\hline Materials & $\begin{array}{l}\text { Elastic modulus } \\
(\mathrm{GPa})\end{array}$ & Poisson's ratio & $\begin{array}{l}\text { Fracture energy } \\
(\mathrm{N} / \mathrm{mm})\end{array}$ & $\begin{array}{l}\text { Tensile strength } \\
(\mathrm{MPa})\end{array}$ & $\begin{array}{l}\text { Adaptive criterion } \\
(\mathrm{MPa})\end{array}$ \\
\hline Concrete & $E_{C}=27.3$ & $v_{C}=0.20$ & - & - & $F t_{\text {adap }}=2.0$ \\
Coarse aggregate & $E_{a g g}=40.0$ & $v_{a g g}=0.20$ & - & - & - \\
Mortar matrix & $E_{m}=20.0$ & $v_{m}=0.20$ & - & - & - \\
Matrix-matrix interface & $E_{m i}=20.0$ & $v_{m i}=0$ & $G_{f_{m i}}=0.14$ & $f_{t_{m i}}=3.0$ & - \\
ITZ & $E_{i t z}=20.0$ & $v_{i t z}=0$ & $G_{f_{i t z}}=0.07$ & $f_{t_{i t z}}=2.0$ & - \\
\hline
\end{tabular}

model (AMS) against the ones from a full mesoscale model (DNS-M). Fig. 5(a) illustrates the geometry, boundary conditions and the initial 800 three-noded triangular FE macroscopic mesh (i.e. coarser scale). The same 25,525 three-noded triangular FE mesoscopic mesh was adopted in both models (Fig. 5(b)). For this finer scale, a total volume fraction of coarse aggregates of $45 \%$ was assumed with their diameters varying between $4 \mathrm{~mm}$ and $8 \mathrm{~mm}$. The aggregates were randomly generated and positioned as proposed by [26].

The material parameters adopted for the homogenized concrete and the three phases of the mesoscopic scale are summarized in Table 2.

This example was strategically selected to validate the mesh adaptive criterion of the multiscale model since a uniform stress state is imposed and the region to be replaced by the mesoscopic mesh is known in advance. The nonlinear analyses are conducted imposing a prescribed horizontal displacement of $0.12 \mathrm{~mm}$ in 600 steps at the right side of the sample (Fig. 5(a)). The behavior of the multiscale model is described by means of the macroscopic mesh with effective material properties $\left(E_{C}\right.$ and $\left.v_{C}\right)$ from the 1st to 64th load step. At the 64th step, the adaptive mesh criterion is reached $\left(\sigma_{\max }=F t_{\text {adap }}=2.0 \mathrm{MPa}\right.$ ) and the macroscopic mesh (Fig. 5(a)) is totally replaced by the mesoscopic one because all the macroscopic finite elements are deactivated at the same time (Fig. 5(b)). From this point on, the multiscale model is represented by the mesoscopic mesh and the crack propagation process is simulated by the mesh fragmentation technique. A numerical analysis using a full mesoscale model from the 1st step was conducted as well.

In Fig. 6 the reaction force is plotted against the imposed displacement for the two models considered. Note that the 64th load step is indicated (i.e. arrow) to show the moment at which the mesh of the multiscale model is enhanced. To validate the numerical model, the results in terms of crack propagation were also compared for the final load stage, as depicted in Fig. 7. 


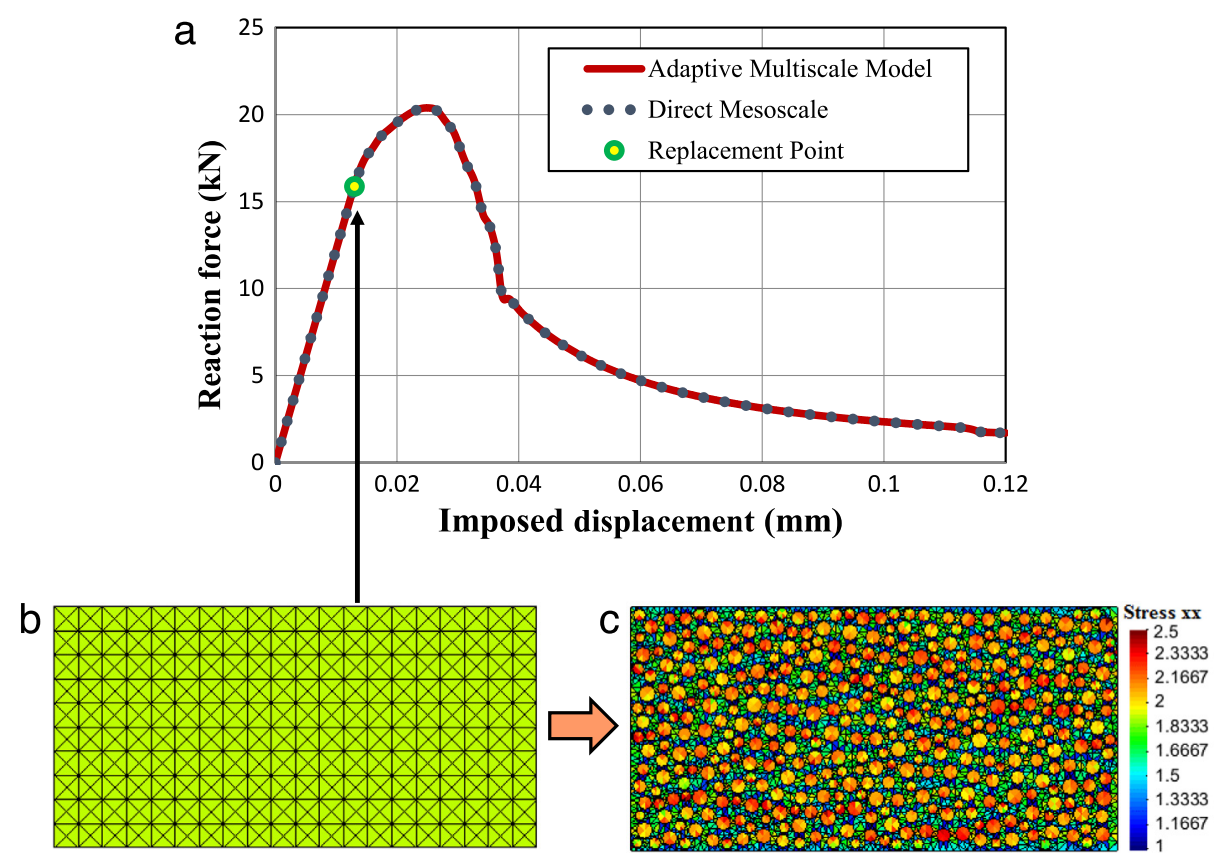

Fig. 6. Reaction force against imposed displacement curves for both models: adaptive concurrent multiscale model (AMS) and full mesoscale model (DNS-M).

\section{a}
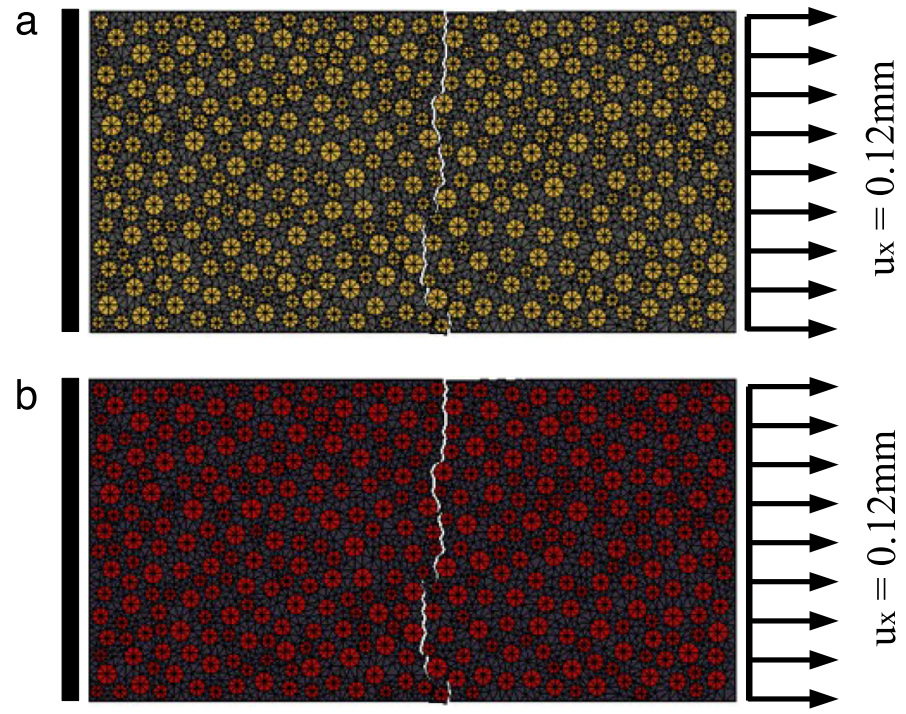

Fig. 7. Failure pattern obtained at final load: (a) adaptive concurrent multiscale model (AMS); and (b) full mesoscale model (DNS-M).

\subsection{L-shaped panel}

In this second example the panel tested experimentally by [47] at the University of Innsbruck was numerically analyzed using the adaptive concurrent multiscale simulation (AMS). To compare the AMS performance against others well-known approaches, two additional analyses were also conducted based on the full mesoscale model (DNS$\mathrm{M})$ and the concurrent multiscale model (DMS). For the three cases, plane stress state conditions with an out of plane thickness of $100 \mathrm{~mm}$ were assumed. 


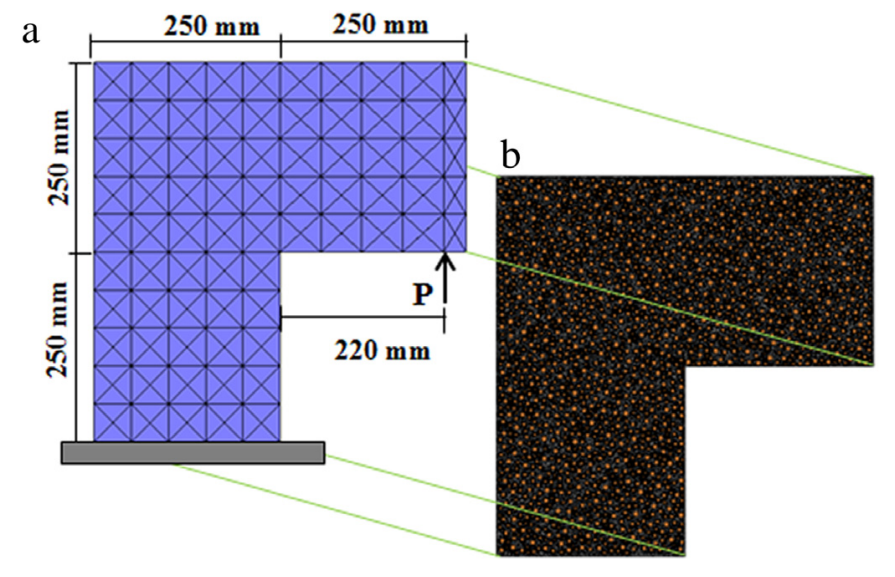

Fig. 8. L-shaped panel: (a) test setup and macroscopic mesh and (b) mesoscopic mesh.

Table 3

Material parameters for the L-shaped panel.

\begin{tabular}{llllll}
\hline Materials & $\begin{array}{l}\text { Elastic modulus } \\
(\mathrm{GPa})\end{array}$ & Poisson's ratio & $\begin{array}{l}\text { Fracture energy } \\
(\mathrm{N} / \mathrm{mm})\end{array}$ & $\begin{array}{l}\text { Tensile strength } \\
(\mathrm{MPa})\end{array}$ & $\begin{array}{l}\text { Adaptive criteria } \\
(\mathrm{MPa})\end{array}$ \\
\hline Concrete & $E_{C}=20.0$ & $v_{C}=0.18$ & - & - & $F t_{a d a p}=2.0$ \\
Coarse aggregate & $E_{a g g}=37.0$ & $v_{a g g}=0.18$ & - & - & - \\
Mortar matrix & $E_{m}=18.5$ & $v_{m}=0.18$ & - & - & - \\
Matrix-matrix interface & $E_{m i}=18.5$ & $v_{m i}=0$ & $G_{f_{m i}}=0.14$ & $f_{t_{m i}}=2.6$ & - \\
ITZ & $E_{i t z}=18.5$ & $v_{i t z}=0$ & $G_{f_{i t z}}=0.07$ & $f_{t_{i t z}}=1.3$ & - \\
\hline
\end{tabular}

Fig. 8(a) shows the test setup and the macroscopic mesh consisting of 300 three-noded triangular finite elements adopted for the first layer of the AMS (as depicted in Fig. 1). The lower horizontal edge is fixed and a vertical load is applied at a distance of $30 \mathrm{~mm}$ from the vertical right side. The mesoscopic mesh is based on the mesoscale model proposed by [26]. The coarse aggregate shapes are approximated by regular octagons which are randomly generated by using the "take-and-place" concept [2] and distributed based on the Fuller's curve with a volume fraction of 20,7\% (Fig. 8(b)). The aggregate diameter varies from $2 \mathrm{~mm}$ to $8 \mathrm{~mm}$, as described by the experiments. The finite element meshes employed in the DNS-M and DMS are composed of 161,013 and 65,188 three-noded triangular finite elements, respectively.

Table 3 lists the adopted material parameters, which correspond to the same ones assumed by [25]. They were obtained based on the experimental results and are in line with the parameters adopted in previous studies dealing with the same experiment and achieving very satisfactory results in terms of both, crack propagation and macro structural response $[48,49]$. In the mesoscale modeling of the concrete, the value of the tensile strength adopted to the matrix-matrix interface is twice the value adopted for the ITZ, as described in detail in [26]. Furthermore, as the adaptive indicator is a value between the strengths of the ITZ and the mortar matrix, a number of initially cracks (microcracks) are simultaneously formed throughout the ITZs at the moment that the mesoscopic mesh is activated.

Fig. 9 shows the crack propagation process for six loading stages (i.e. step-loads 50, 100, 150, 200, 250, 500). The crack initiates at the inner corner (Fig. 9 (a)) and propagates toward the other side of the L-shaped specimen, activating the mesoscale progressively. Details of microcracks being formed during the first load stages in the ITZs can be seen in the zoomed Fig. 9(a) and (b) (both with scaling factor of 300).

The numerical results are compared against the experimental ones, in terms of both, crack propagation process and applied load versus displacement curve, as shown in Fig. 10. The numerical and experimental results are in very good agreement, indicating the ability of the proposed approach to predict the crack formation and propagation in composite materials like concrete.

The results were also compared using the results from DMS and DNS-M analyses. The crack propagation process and the applied load $x$ displacement curves are illustrated in Figs. 11 and 12, respectively. The predominant crack 
a
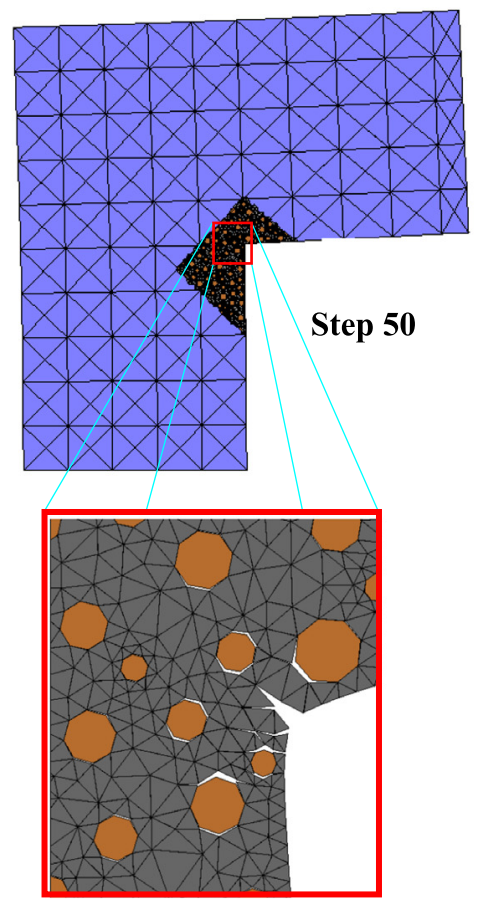

c

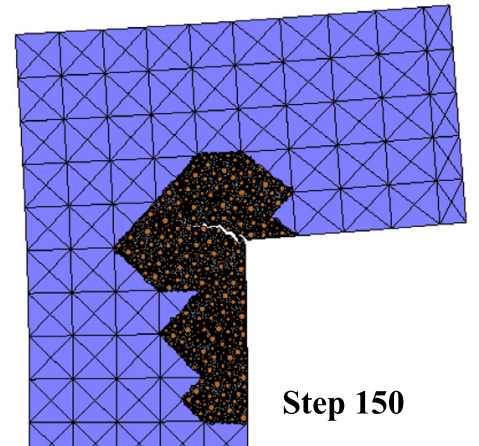

e

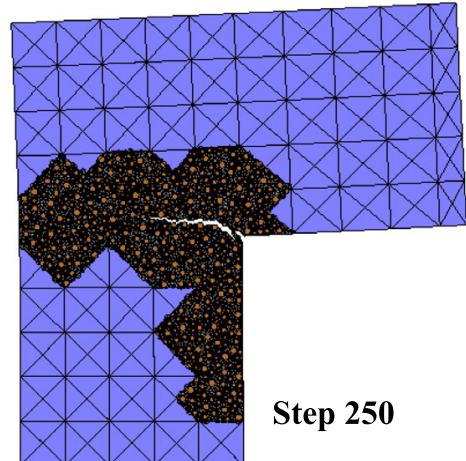

b

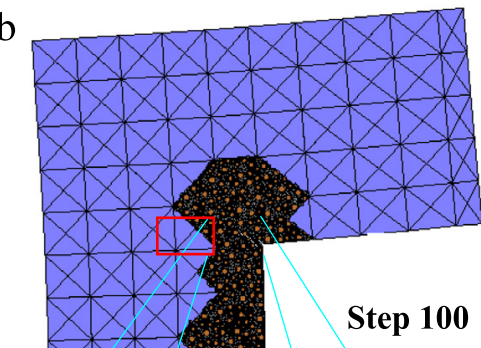

Step 100

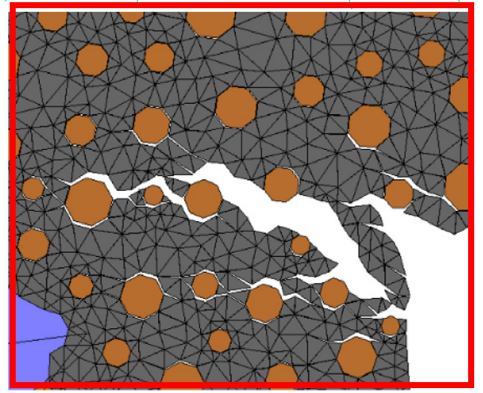

d

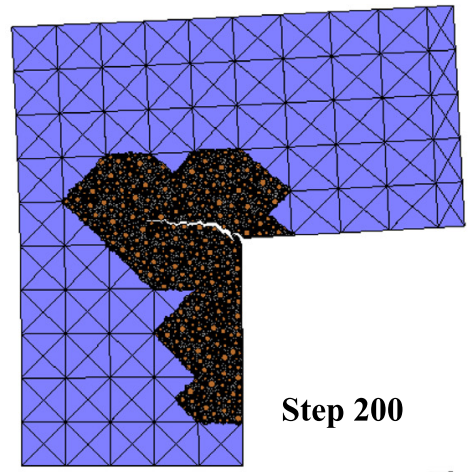

f

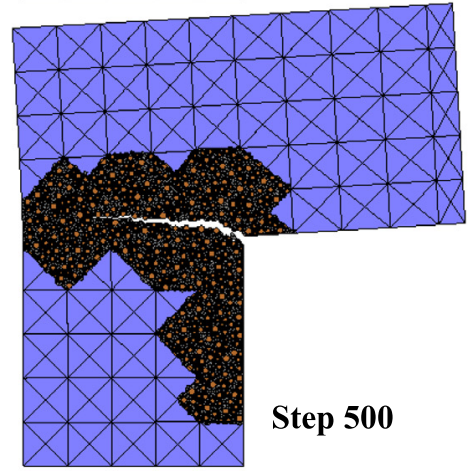

Fig. 9. Crack propagation process: (a) stage I: step-load 50 (scaling factor of 150); (b) stage II: step-load 100 (scaling factor of 150); (c) stage III: step-load 150 (scaling factor of 100); (d) stage IV: step-load 200 (scaling factor of 50), stage V: step-load 250 (scaling factor of 50); and (f) stage VI: step-load 500 (scaling factor of 50). 

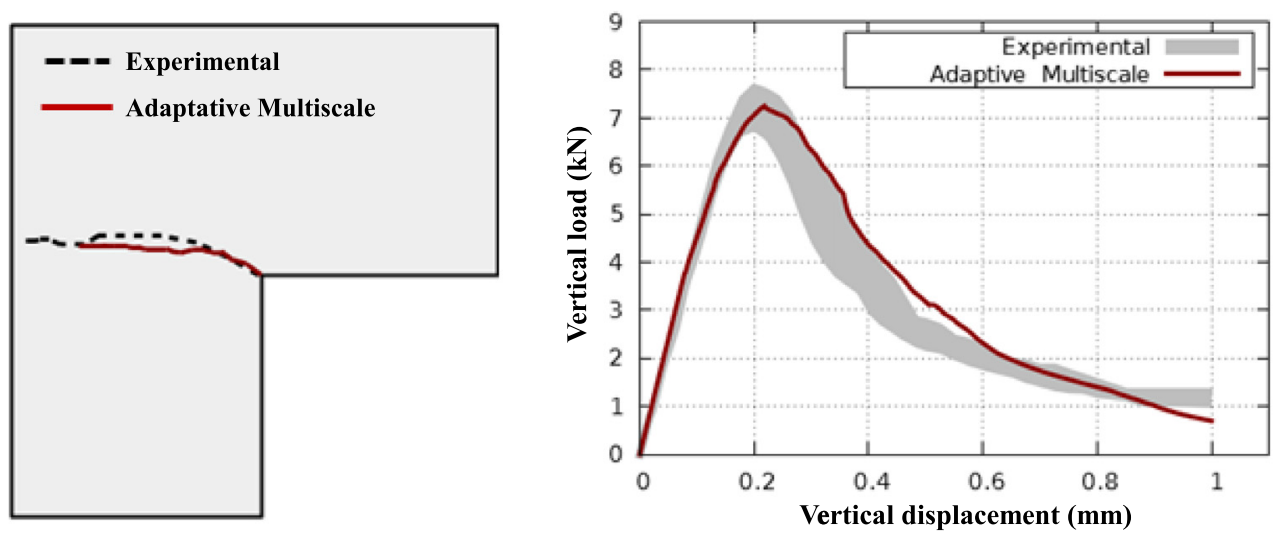

Fig. 10. L-shaped panel results: (a) experimental observations against numerical crack pattern; and (b) load versus displacement curves.
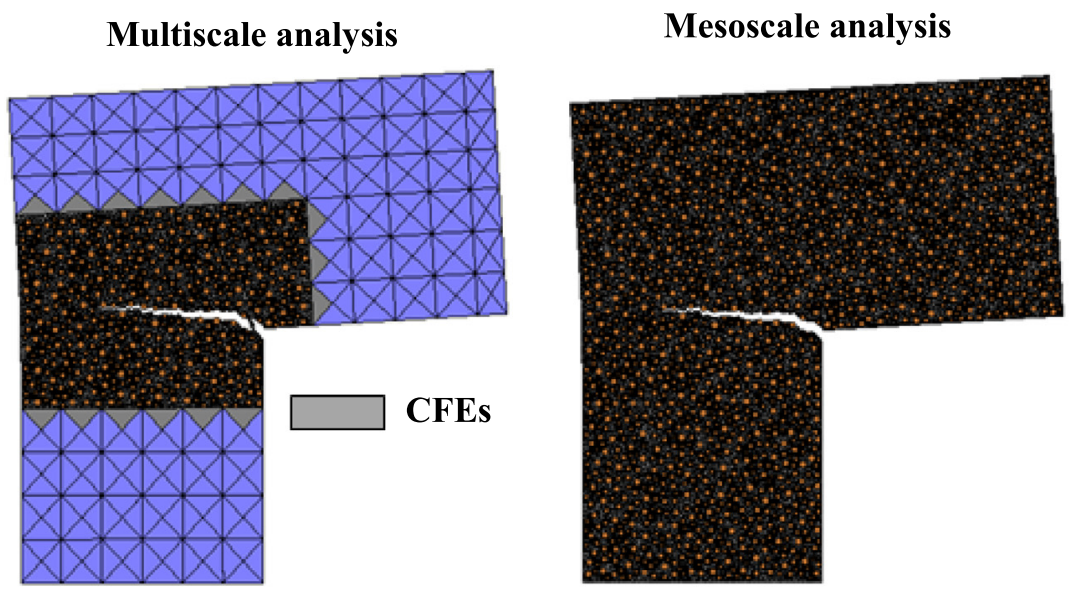

Fig. 11. Deformed state of the L-shaped panel (scaling factor of 50): (a) DMS; and (b) DNS-M.

paths obtained with the DMS (Fig. 11(a)) and DNS-M (Fig. 11(b)) are very similar to the one obtained with the AMS (Fig. 9(f)). In addition, the agreement between the structural curves depicted in Fig. 12 is remarkable. The slight differences between them could be associated with the distinct mesh in each strategy.

Considering the size of the system of equations to be solved, it is anticipated that the computational performance of the AMS is more efficient than the DMS and DNS-M. Fig. 13 presents the number of active degrees of freedom during the analysis for each type of model, which is an indication of the computational effort demanded by the different methods, showing that the Adaptive Multiscale is the more efficient one in this respect.

\subsection{Four-point bending of notched beam}

This example is aimed at challenging the ability of the proposed adaptive multiscale technique to predict the cracking process observed in the concrete beam with a notched, as reported in Galvez et al. (1998). Fig. 14(a) shows the geometry of the beam alongside with the macroscopic mesh (594 elements) and the imposed boundary conditions. Fig. 14(b) presents the mesoscopic mesh (30,376 elements). The out-of-plane thickness is $50 \mathrm{~mm}$. As the grading curve of the aggregates was not reported (Galvez et al., 1998), the Fuller's curve was assumed with a percentage of aggregates of $40 \%$, with a minimum diameter of $3 \mathrm{~mm}$ and maximum diameter of $5 \mathrm{~mm}$.

The material properties adopted for the macroscale and mesoscale constituents are listed in Table 4. Only the homogeneous (i.e. global or macro) properties of the concrete, like Young's modulus $\left(E_{c}=38.0 \mathrm{GPa}\right)$, tensile strength 


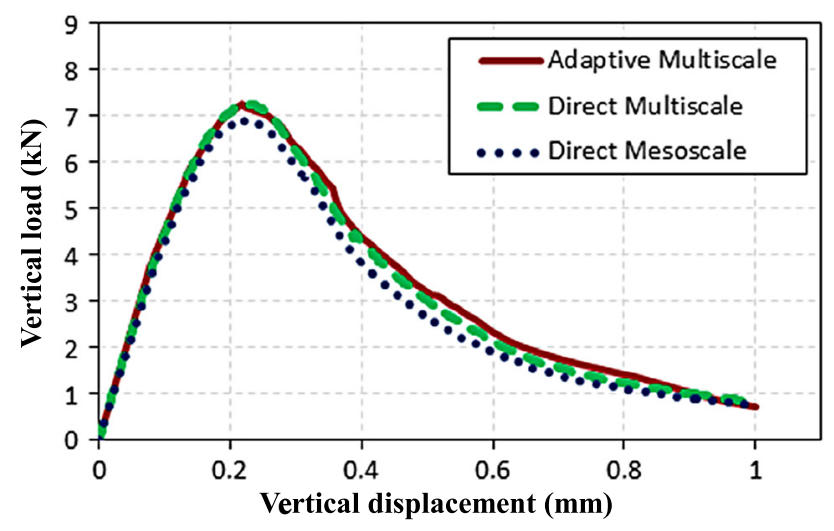

Fig. 12. Load against displacement curves for the AMS, DMS and DNS-M.

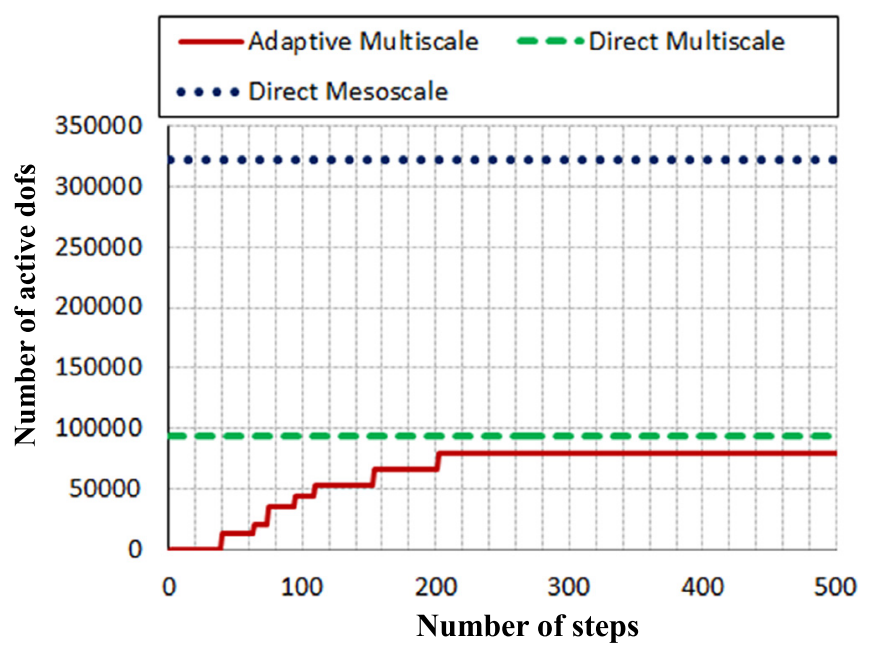

Fig. 13. Number of active degrees of freedom against number of steps.

$\left(f_{t_{c}}=3.0 \mathrm{MPa}\right)$ and the fracture energy $\left(G_{f_{c}}=0.069 \mathrm{~N} / \mathrm{mm}\right)$, were reported in Galvez et al. (1998). To estimate the mesoscale parameters the method proposed by Rodrigues et al. (2016) was followed in this work, as briefly explained next. The Young's modulus of the mortar matrix was estimated from an inverse analysis based on the rule of mixture proposed by [50] and assuming an aggregate Young's modulus of 50.0 GPa. The same Young's modulus was assumed for the matrix-matrix interface elements. The tensile strength and fracture energy of the matrix-matrix interface elements and ITZ interface elements were calculated so that the average values are equivalent to the ones reported in the experiments, they also keep a ratio of 2 between them (i.e. $\left(f_{t_{m i}}+f_{t_{i t z}}\right) / 2=f_{t_{c}}$ with $f_{t_{m i}} / f_{t_{i t z}}=2$ and $\left(G_{f_{m i}}+G_{f_{i t z}}\right) / 2=G_{f_{c}}$ with $\left.G_{f_{m i}} / G_{f_{i t z}}=2\right)$.

The analysis was conducted under plane stress condition, in 1000 incremental load steps, controlling the displacement of the loaded point. Fig. 15 illustrates the macroscopic mesh being progressively replaced by the mesoscopic one, at the same time that the main crack propagates from the notch to the point of load application. Note that, at the bottom of the beam some mesoscopic elements were also activated. However, the main crack propagation reduces the tension stress in this region, stopping the adaptive process in this part of the beam.

Fig. 16 compares the predicted crack path against the experimental scattered response. The predicted crack follows a path that falls inside the observed cracked zone, with a zigzag-type propagation induced by the weak ITZ surrounding the stiff aggregates. Note that the experimental cracks reported in Fig. 16 are approximated, since they were obtained after the scanning of the original figures. 
a

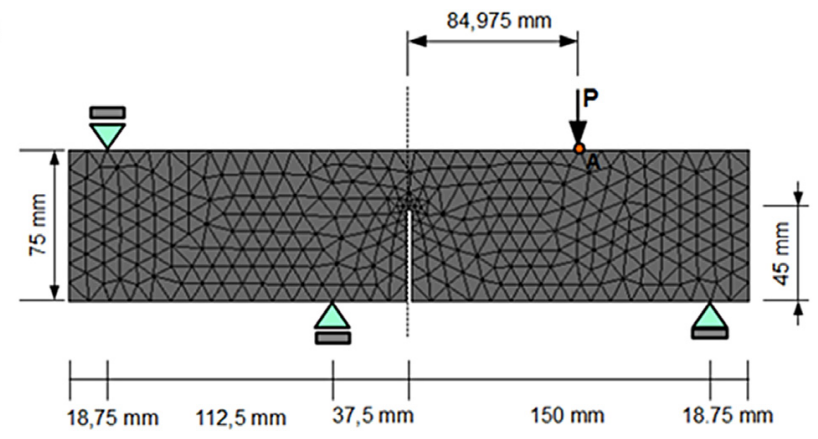

$\mathrm{b}$

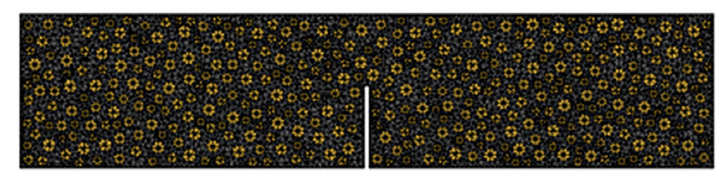

Fig. 14. Four point bending beam: (a) dimensions, macroscopic mesh and boundary conditions and (b) initially deactivated mesoscopic mesh.

Table 4

Material parameters for the four-point bending beam.

\begin{tabular}{llllll}
\hline Materials & $\begin{array}{l}\text { Elastic modulus } \\
(\mathrm{GPa})\end{array}$ & Poisson's ratio & $\begin{array}{l}\text { Fracture energy } \\
(\mathrm{N} / \mathrm{mm})\end{array}$ & $\begin{array}{l}\text { Tensile strength } \\
(\mathrm{MPa})\end{array}$ & $\begin{array}{l}\text { Adaptive criteria } \\
(\mathrm{MPa})\end{array}$ \\
\hline Concrete & $E_{C}=38.0$ & $v_{C}=0.2$ & - & - & $F t_{a d a p}=2.0$ \\
Coarse aggregate & $E_{a g g}=50.0$ & $v_{a g g}=0.2$ & - & - & - \\
Mortar matrix & $E_{m}=30.2$ & $v_{m}=0.2$ & - & - & - \\
Matrix-matrix interface & $E_{m i}=30.2$ & $v_{m i}=0$ & $G_{f_{m i}}=0.092$ & $f_{t_{m i}}=4.0$ & - \\
ITZ & $E_{i t z}=30.2$ & $v_{i t z}=0$ & $G_{f_{i t z}}=0.046$ & $f_{t_{i t z}}=2.0$ & - \\
\hline
\end{tabular}

During the analysis, the mesoscopic mesh is progressively activated, increasing the number of degrees of freedom, as shown in Fig. 17. The structural response in terms of vertical load versus CMOD (i.e. crack mouth opening displacement) is shown in Fig. 18 for both: numerical simulation and the experimental results. The model is in excellent agreement with the observed experimental range. This very satisfactory outcome in terms of structural (or global) behavior demonstrates that the proposed approach is able not only to qualitative reproduce very satisfactorily the observed crack pattern, but it also provides excellent quantitative results.

\section{Conclusions}

This paper presents a novel adaptive concurrent multiscale model for concrete based on the inclusion of coupling finite elements. The proposed adaptive scheme can deal with totally independent meshes associated with distinct material scales. The concrete is initially idealized as homogeneous (i.e. single-phase) material represented by a macroscopic mesh. As loading progresses, the model is gradually enriched in the critical zones with a mesoscopic mesh that includes detailed information about the three phases typically found in concrete, namely: coarse aggregates, mortar matrix and interfacial transition zone. The critical zones are detected by a pre-defined stress-based criterion that dictates the need of a change in scale to properly model the material degradation. The modeling of the concrete internal-structure (i.e. mesoscale) is conducted by means of the mesh fragmentation technique that combines highaspect-ratio elements with a damage mechanical model for a realistic modeling of the crack formation and propagation phenomena. A key component of the proposed approach is the coupling finite elements that allow anchoring (in an efficient and elegant manner), the floating nodes of the evolving mesoscale to the ones of the pre-existing macroscale.

Three challenging numerical examples associated with well-known case studies were adopted to validate the proposed approach. The response and performance of the models were very satisfactory in all the analyzed cases, in terms of both: 
a

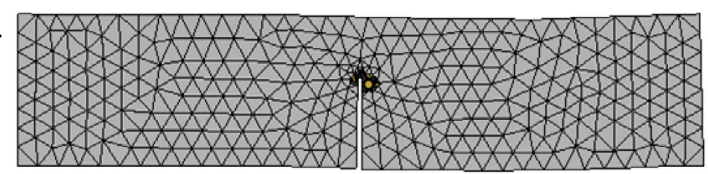

\section{b}

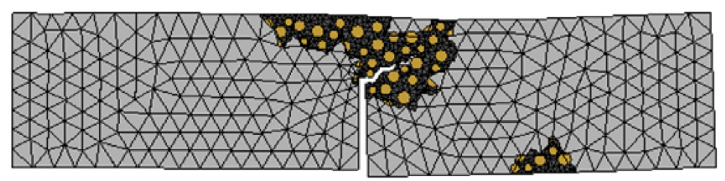

C

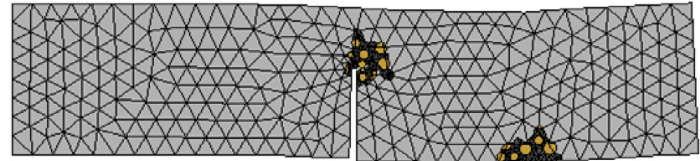

d

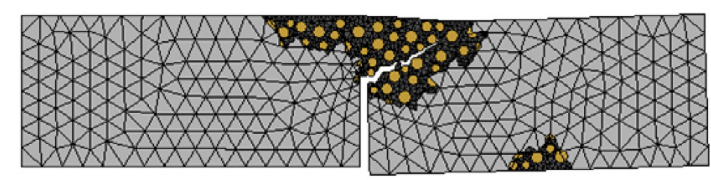

e

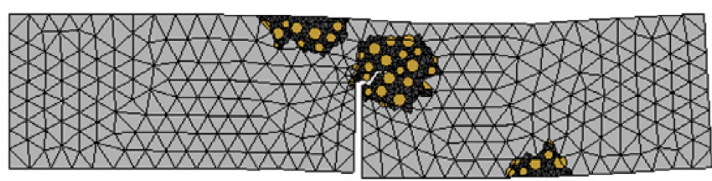

f

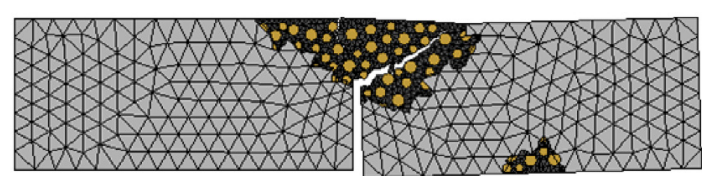

Fig. 15. Deformed configuration of the beam in different stage of load, with scaling factor of 100 .

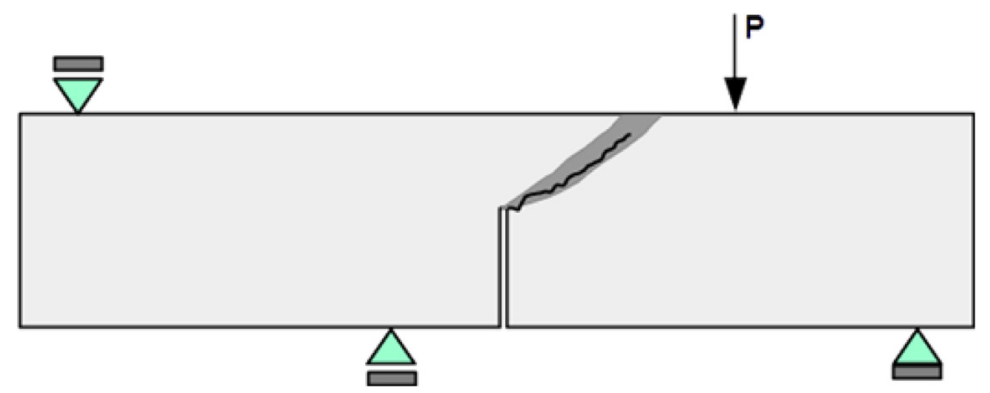

Fig. 16. Experimental crack range response (for four tests) compared and numerical crack pattern.

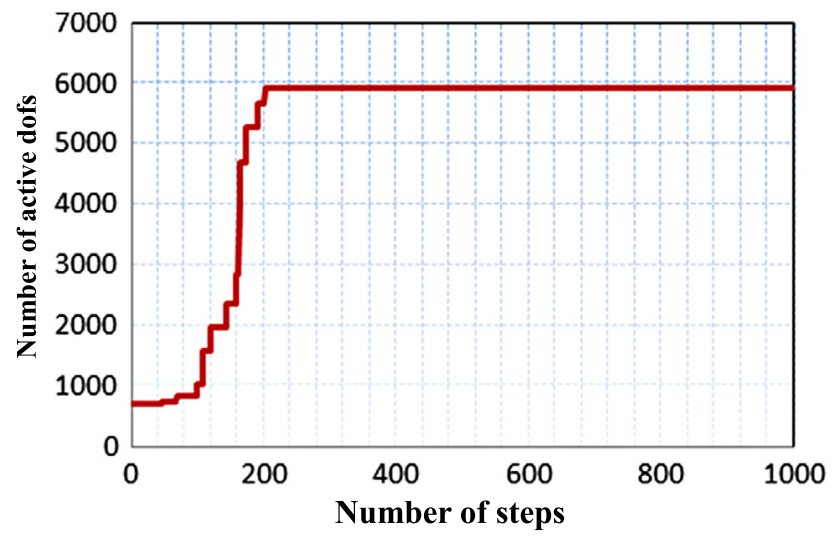

Fig. 17. Number of active dofs (starts with 688 and finishes with 5940 dofs) as function of the analysis steps.

(i) realistic predictions of crack formation and prorogation were obtained, replicating accurately the observed experimental patterns;

(ii) very satisfactory matching between the experimental and modeling results in terms of global-structural (macro) response of the concrete structure. 


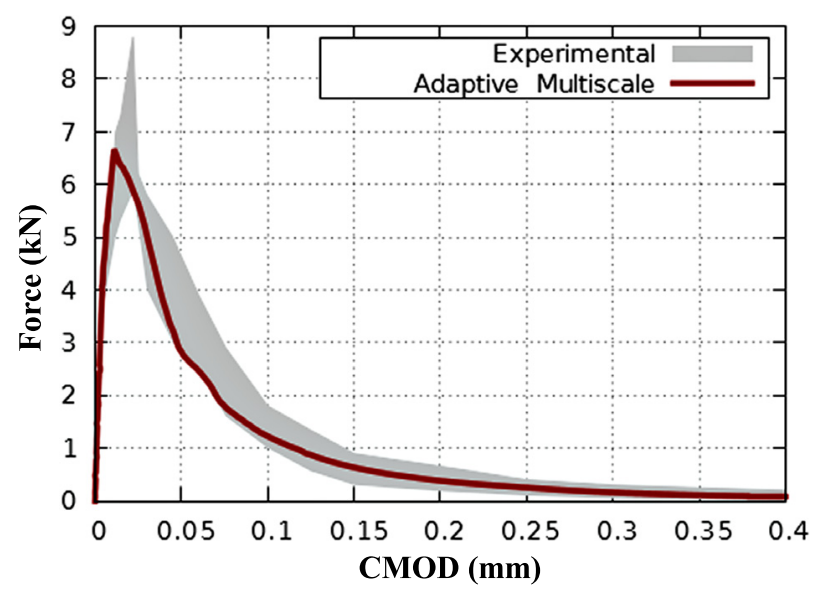

Fig. 18. Load-CMOD curve compared with the experimental range response.

The proposed framework has number of advantages with respect to existing methods. For example, it is able to incorporate a detailed representation of the mesoscale in those critical regions that are actually needed only. This represents a clear advantage with respect to full mesoscopic models that require a very fine discretization in the whole domain and from the beginning of the analysis, with the associated (very high) computational cost. Furthermore, as the mesh enrichment process is driven by a criterion that depends on how stresses develop in the structure, it is not necessary to guess in advance the regions where a denser mesh will be necessary, as it happens in analyses based on the standard concurrent multiscale method. In other words, mesh enhancement and loading processes are concurrent because the proposed technique uses the evolving stress distribution to identify those regions that are prone to be degraded without the need of anticipating/guessing where the mesh refinement will be necessary. Another advantage of this technique is that the coupling finite elements (used to link the non-matching meshes) can be easily implemented in standard FE programs. Besides, this method inherits all the advantages of the mesh fragmentation technique, which is adopted for a detailed modeling of the mesoscale and the phenomena involved in the crack formation and propagation processes. This technique has been already widely validated to model the crack formation and propagation in concrete and soils. Finally, the concrete degradation processes and crack formation are modeled in an entirely continuum approach, without the need of defining special zero technique elements, or similar (with the associated ad-doc interpolation techniques and constitutive models), and without the need of incorporating complex algorithm(s) to properly capture the crack paths. In this approach the crack formation and propagation depend on local stress evolution and material properties only.

It was shown that the proposed method is robust and computationally efficient when compared against other existing ones in problems involving 2D analyses. Advantages will be certainly more relevant when analyzing 3D problems, where a good performance in terms of CPU time is critical for solving actual engineering problems. However, this aspect is out of the scope of this paper and will be addressed in future research.

\section{Acknowledgments}

The authors wish to acknowledge the financial support of the National Council for Scientific and Technological Development (CNPq), Coordination for the Improvement of Higher Education Personnel (CAPES) and São Paulo Research Foundation (FAPESP). The last author would like to particularly acknowledge the financial support from the São Paulo Research Foundation (FAPESP, proc. 2016/19479-2).

\section{References}

[1] Z.P. Bazant, M.R. Tabbara, M.T. Kazemi, G. Pijaudier-Cabot, Random particle model for fracture of aggregate or fiber composites, J. Eng. Mech. 116 (8) (1990) 1686-1705. http://dx.doi.org/10.1061/(ASCE)0733-9399(1990)116:8(1686). 
[2] P. Wriggers, S. Moftah, Mesoscale models for concrete: Homogenisation and damage behaviour, Finite Elem. Anal. Des. 42 (7) (2006) 623636. http://dx.doi.org/10.1016/j.finel.2005.11.008. The Seventeenth Annual Robert J. Melosh Competition. The Seventeenth Annual Robert J. Melosh Competition. http://www.sciencedirect.com/science/article/pii/S0168874X05001563.

[3] J. Fish, K. Shek, Multiscale analysis of composite materials and structures, Compos. Sci. Technol. 60 (12) (2000) $2547-2556$.

[4] S. Ghosh, J. Bai, P. Raghavan, Concurrent multi-level model for damage evolution in microstructurally debonding composites, Mech. Mater. 39 (3) (2007) 241-266.

[5] A. Ibrahimbegović, D. Markovič, Strong coupling methods in multi-phase and multi-scale modeling of inelastic behavior of heterogeneous structures, Comput. Methods Appl. Mech. Engrg. 192 (28) (2003) 3089-3107.

[6] M. Kulkarni, K. Matouš, P. Geubelle, Coupled multi-scale cohesive modeling of failure in heterogeneous adhesives, Internat. J. Numer. Methods Engrg. 84 (8) (2010) 916-946.

[7] V.P. Nguyen, O. Lloberas-Valls, M. Stroeven, L.J. Sluys, Homogenization-based multiscale crack modelling: From micro-diffusive damage to macro-cracks, Comput. Methods Appl. Mech. Engrg. 200 (9) (2011) 1220-1236.

[8] O. Lloberas-Valls, D. Rixen, A. Simone, L. Sluys, Domain decomposition techniques for the efficient modeling of brittle heterogeneous materials, Comput. Methods Appl. Mech. Engrg. 200 (13) (2011) 1577-1590.

[9] O. Lloberas-Valls, D. Rixen, A. Simone, L. Sluys, Multiscale domain decomposition analysis of quasi-brittle heterogeneous materials, Internat. J. Numer. Methods Engrg. 89 (11) (2012) 1337-1366.

[10] S. Toro, P.J. Sánchez, A.E. Huespe, S.M. Giusti, P.J. Blanco, R. Feijóo, A two-scale failure model for heterogeneous materials: Numerical implementation based on the finite element method, Internat. J. Numer. Methods Engrg. 97 (5) (2014) 313-351.

[11] S. Toro, P.J. Sánchez, P.J. Blanco, E. de Souza Neto, A.E. Huespe, R. Feijóo, Multiscale formulation for material failure accounting for cohesive cracks at the macro and micro scales, Int. J. Plast. 76 (2016) 75-110.

[12] J. Oliver, M. Caicedo, E. Roubin, A. Huespe, J. Hernández, Continuum approach to computational multiscale modeling of propagating fracture, Comput. Methods Appl. Mech. Engrg. 294 (2015) 384-427. http://dx.doi.org/10.1016/j.cma.2015.05.012. http://www.sciencedirect. com/science/article/pii/S0045782515001851.

[13] J. Oliver, M. Caicedo, A. Huespe, J. Hernández, E. Roubin, Reduced order modeling strategies for computational multiscale fracture, Comput. Methods Appl. Mech. Engrg. 313 (2017) 560-595.

[14] O. Lloberas-Valls, D. Rixen, A. Simone, L. Sluys, On micro-to-macro connections in domain decomposition multiscale methods, Comput. Methods Appl. Mech. Engrg. 225-228 (0) (2012) 177-196. http://dx.doi.org/10.1016/j.cma.2012.03.022. http://www.sciencedirect.com/ science/article/pii/S0045782512000989.

[15] I.M. Gitman, Representative Volumes and Multi-Scale Modelling of Quasi-Brittle Materials, TU Delft, Delft University of Technology, 2006.

[16] I. Gitman, H. Askes, L. Sluys, Coupled-volume multi-scale modelling of quasi-brittle material, Eur. J. Mech.-A/Solids 27 (3) (2008) $302-327$.

[17] S. Eckardt, Adaptive heterogeneous multiscale models for the nonlinear simulation of concrete, 2009.

[18] V.P. Nguyen, M. Stroeven, L.J. Sluys, Multiscale failure modeling of concrete: Micromechanical modeling, discontinuous homogenization and parallel computations, Comput. Methods Appl. Mech. Engrg. 201 (2012) 139-156.

[19] P.J. Sánchez, P. Blanco, A.E. Huespe, R. Feijóo, Failure-oriented multi-scale variational formulation: Micro-structures with nucleation and evolution of softening bands, Comput. Methods Appl. Mech. Engrg. 257 (2013) 221-247.

[20] S. Nemat-Nasser, M. Hori, Micromechanics: Overall Properties of Heterogeneous Materials, vol. 37, Elsevier, 2013.

[21] E. Landis, J. Bolander, Explicit representation of physical processes in concrete fracture, J. Phys. D: Appl. Phys. 42 (21) (2009) 1-17. http:// stacks.iop.org/0022-3727/42/i=21/a=214002.

[22] F. Feyel, J.-L. Chaboche, FE 2 multiscale approach for modelling the elastoviscoplastic behaviour of long fibre SiC/Ti composite materials, Comput. Methods Appl. Mech. Engrg. 183 (3) (2000) 309-330.

[23] S. Häfner, S. Eckardt, T. Luther, C. Könke, Mesoscale modeling of concrete: Geometry and numerics, Comput. Struct. 84 (7) (2006) $450-461$. http://dx.doi.org/10.1016/j.compstruc.2005.10.003. http://www.sciencedirect.com/science/article/pii/S0045794905003585.

[24] Z. Wang, A. Kwan, H. Chan, Mesoscopic study of concrete I: Generation of random aggregate structure and finite element mesh, Comput. Struct. 70 (5) (1999) 533-544. http://dx.doi.org/10.1016/S0045-7949(98)00177-1. http://www.sciencedirect.com/science/article/ pii/S0045794998001771.

[25] J.F. Unger, S. Eckardt, Multiscale modeling of concrete from mesoscale to macroscale, Arch. Comput. Methods Eng. 18 (3) (2011) $341-393$. http://dx.doi.org/10.1007/s11831-011-9063-8.

[26] E.A. Rodrigues, O.L. Manzoli, L.A. Bitencourt Jr., T.N. Bittencourt, 2D mesoscale model for concrete based on the use of interface element with a high aspect ratio, Int. J. Solids Struct. 94 (2016) 112-124.

[27] G. Cusatis, Z. Bažant, L. Cedolin, Confinement-shear lattice CSL model for fracture propagation in concrete, Comput. Methods Appl. Mech. Engrg. 195 (52) (2006) 7154-7171. http://dx.doi.org/10.1016/j.cma.2005.04.019. Computational Modelling of Concrete. http://www. sciencedirect.com/science/article/pii/S0045782505003956.

[28] X. Du, L. Jin, G. Ma, Numerical modeling tensile failure behavior of concrete at mesoscale using extended finite element method, Int. J. Damage Mech. 23 (7) (2014) 872-898. http://dx.doi.org/10.1177/1056789513516028.

[29] S. Ghosh, K. Lee, P. Raghavan, A multi-level computational model for multi-scale damage analysis in composite and porous materials, Int. J. Solids Struct. 38 (14) (2001) 2335-2385.

[30] F.J. Vernerey, M. Kabiri, An adaptive concurrent multiscale method for microstructured elastic solids, Comput. Methods Appl. Mech. Engrg. 241 (2012) 52-64.

[31] B. Sun, Z. Li, Adaptive image-based method for integrated multi-scale modeling of damage evolution in heterogeneous concrete, Comput. Struct. 152 (2015) 66-81.

[32] B. Sun, X. Wang, Z. Li, Meso-scale image-based modeling of reinforced concrete and adaptive multi-scale analyses on damage evolution in concrete structures, Comput. Mater. Sci. 110 (2015) 39-53. 
[33] B. Sun, Z. Li, Adaptive concurrent three-level multiscale simulation for trans-scale process from material mesodamage to structural failure of concrete structures, Int. J. Damage Mech. (2016). http://dx.doi.org/10.1177/1056789516648371.

[34] B. Guo, I. Babuška, The hp version of the finite element method, Comput. Mech. 1 (1) (1986) 21-41.

[35] B. Wohlmuth, A mortar finite element method using dual spaces for the Lagrange multiplier, SIAM J. Numer. Anal. 38 (3) (2001) 989-1012. http://www.jstor.org/stable/3061996.

[36] H. Dhia, G. Rateau, The arlequin method as a flexible engineering design tool, Internat. J. Numer. Methods Engrg. 62 (11) (2005) 1442-1462. http://dx.doi.org/10.1002/nme.1229.

[37] B. Lamichhane, B. Wohlmuth, Mortar finite elements for interface problems, Computing 72 (3-4) (2004) 333-348. http://dx.doi.org/10.1007/ s00607-003-0062-y.

[38] L.A.G. Bitencourt Jr., O.L. Manzoli, P.G.C. Prazeres, E.A. Rodrigues, T.N. Bittencourt, A coupling technique for non-matching finite element meshes, Comput. Methods Appl. Mech. Engrg. 290 (2015) 19-44. http://dx.doi.org/10.1016/j.cma.2015.02.025. http://www.sciencedirect. com/science/article/pii/S0045782515000870.

[39] O.L. Manzoli, M.A. Maedo, L.A. Bitencourt, E.A. Rodrigues, On the use of finite elements with a high aspect ratio for modeling cracks in quasi-brittle materials, Eng. Fract. Mech. 153 (2016) 151-170.

[40] M. Sánchez, O. Manzoli, L. Guimarães, Modeling 3-D desiccation soil crack networks using a mesh fragmentation technique, Comput. Geotech. 62 (0) (2014) 27-39. http://dx.doi.org/10.1016/j.compgeo.2014.06.009. http://www.sciencedirect.com/science/article/pii/ S0266352X14001177.

[41] O. Manzoli, A. Gamino, E. Rodrigues, G. Claro, Modeling of interfaces in two-dimensional problems using solid finite elements with high aspect ratio, Comput. Struct. 94-95 (0) (2012) 70-82. http://dx.doi.org/10.1016/j.compstruc.2011.12.001. http://www.sciencedirect. com/science/article/pii/S0045794911002938.

[42] J. Oliver, M. Cervera, O.L. Manzoli, Strong discontinuities and continuum plasticity models: the strong discontinuity approach, Int. J. Plasticity 15 (3) (1999) 319-351.

[43] J. Oliver, On the discrete constitutive models induced by strong discontinuity kinematics and continuum constitutive equations, Int. J. Solids Struct. 37 (48-50) (2000) 7207-7229. http://dx.doi.org/10.1016/S0020-7683(00)00196-7. http://www.sciencedirect.com/science/article/pii/ S0020768300001967.

[44] J. Oliver, A.E. Huespe, Continuum approach to material failure in strong discontinuity settings., Comput. Methods Appl. Mech. Engrg. 193 (30-32) (2004) 3195-3220.

[45] J. Oliver, A. Huespe, J. Cante, An implicit/explicit integration scheme to increase computability of non-linear material and contact/friction problems, Comput. Methods Appl. Mech. Engrg. 197 (21-24) (2008) 1865-1889. http://dx.doi.org/10.1016/j.cma.2007.11.027. http://www. sciencedirect.com/science/article/pii/S0045782507004756.

[46] P.G.C. Prazeres, L.A.G. Bitencourt Jr., T.N. Bittencourt, O.L. Manzoli, A modified implicit-explicit integration scheme: An application to elastoplasticity problems, J. Braz. Soc. Mech. Sci. Eng. (2015) 1-11. http://dx.doi.org/10.1007/s40430-015-0343-3.

[47] B. Winkler, G. Hofstetter, G. Niederwanger, Experimental verification of a constitutive model for concrete cracking, Proc. Inst. Mech. Eng. L: J. Mater. Design Appl. 215 (2) (2001) 75-86.

[48] P. Dumstorff, G. Meschke, Crack propagation criteria in the framework of X-FEM-based structural analyses, Int. J. Numer. Anal. Methods Geomech. 31 (2) (2007) 239.

[49] N. Labanda, S. Giusti, B. Luccioni, Meso-scale fracture simulation using an augmented lagrangian approach, Int. J. Damage Mech. (2016). http://dx.doi.org/10.1177/1056789516671092.

[50] U.J. Counto, The effect of the elastic modulus of the aggregate on the elastic modulus, creep and creep recovery of concrete, Mag. Concr. Res. 16 (48) (1964) 129-138. 